Delft University of Technology

\title{
Perceptual Eigenmode Distortion Analysis for Motion Cueing Evaluation in Fixed-Wing Aircraft Simulators
}

Tillema, G.H.J.; Stroosma, O.; Miletović, I.; Mulder, Max

\section{DOI}

10.2514/6.2021-1012

\section{Publication date}

2021

Document Version

Final published version

Published in

AIAA Scitech 2021 Forum

\section{Citation (APA)}

Tillema, G. H. J., Stroosma, O., Miletović, I., \& Mulder, M. (2021). Perceptual Eigenmode Distortion Analysis for Motion Cueing Evaluation in Fixed-Wing Aircraft Simulators. In AlAA Scitech 2021 Forum: 11-15 \& 19-21 January 2021Virtual/online event [AIAA 2021-1012] American Institute of Aeronautics and Astronautics Inc. (AIAA). https://doi.org/10.2514/6.2021-1012

Important note

To cite this publication, please use the final published version (if applicable).

Please check the document version above.

\footnotetext{
Copyright

Other than for strictly personal use, it is not permitted to download, forward or distribute the text or part of it, without the consent of the author(s) and/or copyright holder(s), unless the work is under an open content license such as Creative Commons.

Takedown policy

Please contact us and provide details if you believe this document breaches copyrights.

We will remove access to the work immediately and investigate your claim.
} 


\title{
Perceptual Eigenmode Distortion Analysis for Motion Cueing Evaluation in Fixed-Wing Aircraft Simulators
}

\author{
G.H.J. Tillema*, O. Stroosma ${ }^{\dagger}$, I. Miletović ${ }^{\dagger}$ and M. Mulder ${ }^{\S}$
}

The Perceptual Eigenmode Distortion (PEMD), an extension to the Eigenmode Distortion (EMD), is a method for objectively evaluating simulator motion fidelity, developed over the last few years. EMD assesses how the Motion Cueing Algorithm (MCA) distorts the vehicle's perceived eigenmodes. In this paper, EMD is extended by a human perception model, which helps to balance the various motion cue contributions in a more human-centered context. Additionally, a new automatic MCA tuning approach is introduced to create an MCA parameter set that is optimal in terms of eigenmode distortion. The method is applied to a combined linear model of the Cessna Citation 500 for asymmetrical flight and the Classical Washout Algorithm (CWA). A pilot-in-the-loop experiment was performed, with six pilots in the SIMONA Research Simulator, to compare the PEMD method's parameter set with sets designed with the current state-of-the-art method of the Objective Motion Cueing Test (OMCT), and with a baseline motion configuration, as well as a condition without any simulator motion. Throughout each run of the double-blind pairwise comparisons, the Dutch roll eigenmode was externally excited with a gust of semi-random amplitude and direction. Two hypotheses were tested using subjective preferences and through measuring the Dutch roll suppression performance. Subjective preferences varied between and within pilots, and similar results for PEMD and OMCT were found. A significant improvement in performance was found, however, between the no-motion condition and the PEMD. Although the perceived differences between a PEMD-tuned and alternative MCA settings seem very subtle, the improved mode suppression performance suggests the method having merit in flight scenarios where the aircraft's dynamic modes play an important role.

$\begin{array}{lll}\square & =\text { Generic symbol placeholder } \\ \alpha & =\text { Angle-of-attack } \\ \delta_{a} & =\text { Aileron deflection } \\ \delta_{r} & =\text { Rudder deflection } \\ \lambda_{\square} & =\text { Eigenvalue } \\ \omega_{\square} & =\text { Break frequency of filter in CWA channel } \\ \phi, \theta, \psi & =\text { Roll, pitch and yaw angle } \\ \zeta_{\square} & =\text { Damping ratio of CWA channel } \\ \square & =\text { Quantity in perception thresholds } \\ \dot{\square} & =\text { Quantity derivative } \\ \square_{0} & =\text { Quantity trim point } \\ \square & =\text { Quantity augmented vehicle system } \\ \square \\ \square C A & =\text { Quantity in simulator } \\ A^{\square} & =\text { System matrix } \\ a_{\square} & =\text { Actuator accelerations } \\ a_{\square} & =\text { Acceleration in subscripted axis } \\ B^{\square} & =\text { Input matrix } \\ B_{\square} & =\text { Actuator buffer } \\ C^{\square} & =\text { Output matrix } \\ C^{\square} & =\text { Eigenmode cost function } \\ C_{\square} & =\text { Force or moment coefficient } \\ D^{\square} & =\text { Feedthrough matrix } \\ D_{\square}^{\square} & =\text { Euclidean distance } \\ & \end{array}$

$\begin{array}{ll}f_{y} & =\text { Sway specific force } \\ g & =\text { Gravitational acceleration } \\ I_{\square} & =\text { Vehicle moments of inertia } \\ K_{\square} & =\text { Gain of subscripted CWA channel } \\ L_{\square}, M_{\square} & =\text { Non-dimensional moment derivatives } \\ \boldsymbol{l}_{\square} & =\text { Actuator lengths } \\ l_{\square} & =\text { Pilot station moment arms } \\ \boldsymbol{m} & =\text { Representative manoeuvre } \\ \boldsymbol{p} & =\text { MCA parameters } \\ p, r & =\text { Roll and yaw rate } \\ \boldsymbol{u}^{\square} & =\text { Input vector } \\ u, v, w & =\text { Longitudinal, lateral and vertical velocity } \\ V_{\square} & =\text { Matrix of right eigenvectors } \\ \boldsymbol{v}_{\square} & =\text { Actuator velocities } \\ W_{\square}^{\square} & =\text { Weight of eigenmode motion cue } \\ x, y, z & =\text { Axes of reference frame } \\ \boldsymbol{x}_{\square}^{\square} & =\text { Eigenvector } \\ \boldsymbol{x}^{\square} & =\text { State vector } \\ Y_{\square} & =\text { Non-dimensional lateral force derivative } \\ \boldsymbol{y}^{\square} & =\text { Output vector } \\ Z & =\text { Objective function } \\ \boldsymbol{z} & =\text { Modal state }\end{array}$

*MSc. student, Control \& Simulation section, Faculty of Aerospace Engineering, P.O. Box 5058, 2600GB Delft, The Netherlands, e-mail: guido.tillema@nlr.nl

'Researcher, Control \& Simulation section, Faculty of Aerospace Engineering, P.O. Box 5058, 2600GB Delft, The Netherlands, e-mail: o.stroosma@tudelft.nl Senior Member AIAA

${ }^{\dagger}$ Researcher, Control \& Simulation section, Faculty of Aerospace Engineering, P.O. Box 5058, 2600GB Delft, The Netherlands, e-mail: i.miletovic@ tudelft.nl

${ }^{8}$ Professor, Control \& Simulation section, Faculty of Aerospace Engineering, P.O. Box 5058, 2600GB Delft, The Netherlands, e-mail: m.mulder@tudelft.nl Associate Fellow AIAA 


\section{Introduction}

Full flight simulators are standalone devices to replicate the rotations and forces as would be perceived by the pilot in an aircraft. Ideally, the perceived rotations and forces in the flight simulator are identical to the real aircraft. However, where an aircraft has an (almost) infinite motion space, flight simulators have physical limitations.

A Motion Cueing Algorithm (MCA) is needed to deal with these physical limits. It is a component between the flight model (i.e. Equations of Motion (EOM)) and the motion platform [1]. An MCA ensures that the simulated motion platform does not exceed its limits (e.g. actuator lengths), while still providing the pilot with meaningful cues.

The MCA has traditionally mostly been tuned subjectively by MCA-tuning experts, who tuned the variables of the algorithm until the pilot was satisfied. The resulting performance was generally expressed in terms of fidelity, whereby high-fidelity means a high match between simulator and aircraft. As this fidelity rating was subjective, resulting tuning variables were different per pilot, but also dependent on the task and workload of the pilot [2].

The undesirability of only subjective evaluations was already recognized in the 1970s and improved objective evaluations have been developed since, such as the methods by Sinacori [3], Schroeder [4] and Gouverneur [5].

These developments resulted in the current state-of-art method: the Objective Motion Cueing Test (OMCT) [6]. This method has improved the tuning process and gives an objective and detailed picture of the behavior of the MCA and motion platform combined. The OMCT analysis is performed in the frequency domain where it determines a frequency response function providing magnitude- and phase distortions introduced by the MCA and motion platform at a set of 12 discrete frequencies.

Despite the power of OMCT, this method also has its shortcomings, such as its sine-wave input signals not representing realistic motion experienced during flight. Also vehicle dynamics are not directly evaluated in this test. Moreover, within OMCT, each signal is studied in isolation, while aircraft motions are linked through the vehicle dynamics. Any non-linearities and cross-couplings in the MCA can yield behavior that is not reliably captured by OMCT's frequency response function[7].

To tackle these shortcomings, the Control \& Simulation section at the Faculty of Aerospace Engineering in TU Delft has developed a novel objective evaluation method, the Eigenmode Distortion (EMD) [8]. The EMD method uses a different approach to evaluate objective fidelity of the combined aircraft model and motion cueing algorithm. Rather than analyzing in the frequency domain, this method investigates distortions of motion cues imposed by the MCA in terms of eigenvectors, which represent the dynamic modes of a vehicle linear model. This was achieved by combining linearized vehicle dynamics and a linearized MCA, into a single combined linear time-invariant system. Initially, EMD was developed for and applied to rotorcraft simulators [7]. Recent work of Stoev et al. [9] has extended EMD to fixed-wing aircraft simulations and applied it to a linear model of the Cessna Citation 500 for symmetric flight in combination with the Classical Washout Algorithm (CWA) [1] as the MCA.

The purpose of this study is to improve the EMD method by incorporating a human perception model, yielding the Perceptual Eigenmode Distortion (PEMD) method. With PEMD, the contributions of individual motion cues to a pilot's perception of a mode can be better identified. In this paper, EMD is applied to a lateral model of the Cessna Citation 500 for asymmetrical flight and the CWA is used as the MCA. Besides, a new automatic tuning approach is introduced and a pilot-in-the-loop experiment is conducted on the SIMONA Research Simulator (SRS) [10] at the Delft University of Technology, to evaluate the OMCT and PEMD methods, using both subjective and objective metrics. The objective metric used for this experiment has been designed in response to the pilot opinions in Stoev's pilot-in-the-loop experiment being hampered by inconsistencies, both between- and within subjects. A more objective metric is hoped to provide more insight into the method's value.

This paper is structured as follows; the EMD and its implementation to the lateral model of the Cessna Citation 500 is introduced in Section [I] The EMD pilot perception model addition is explained in Section III] yielding PEMD. The PEMD analysis is then used to derive a tuning algorithm in Section IV. The potential of PEMD is evaluated with the experiment discussed in Section $\mathrm{V}$. The results of this experiment are given in Section $\mathrm{VI}$ Lastly, a discussion, recommendation and conclusion is given in Section VII] VIII and IX, respectively. 


\section{Eigenmode Distortion}

\section{A. Methodology}

Despite the power of OMCT, which, at the time of writing, is the state-of-the-art method for evaluating objective motion cueing fidelity, the method has its shortcomings. OMCT captures the performance of the MCA and motion platform combined, however the simulated vehicle dynamics are not directly included. Furthermore, the simulated response to each signal is studied in isolation, while aircraft motions are linked through dynamics. It was shown that several intricate interactions between aircraft- and MCA dynamics could therefore not be captured [7].

The EMD method, developed by Miletović [8], is an objective evaluation method designed to address these shortcomings. EMD investigates the distortion of motion cues, imposed by the MCA, in terms of the vehicle's eigenmodes. Whereas eigenvalues provide information about the nature of an eigenmode, eigenvectors provide information on how different states (e.g. surge and pitch) interact in each eigenmode in terms of phase and magnitude. To a human pilot, the eigenvector represents "what the mode feels like". Unfortunately most MCAs do not pass through these eigenvectors undistorted to the motion system, decreasing simulator fidelity.

To determine these motion cue distortions, a combined linear model of the flight model (i.e. the Equations of Motion $(\mathrm{EOM}))$ and MCA is used. The linear model structure for the EOM in asymmetric flight is given in Equations 1 to 3 . These EOM describe the dynamic behaviour of the aircraft and represent the states underlying the cues experienced by the pilot in the aircraft.

$$
\begin{aligned}
\dot{\boldsymbol{x}}^{\text {EOM }} & =A^{\text {EOM }} \boldsymbol{x}^{\text {EOM }}+B^{\text {EOM }} \boldsymbol{u}^{\text {EOM }} \\
\boldsymbol{x}^{\text {EOM }} & =\left[\begin{array}{llll}
v & \phi & p & r
\end{array}\right]^{T} \\
\boldsymbol{u}^{\text {EOM }} & =\left[\begin{array}{ll}
\delta_{a} & \delta_{r}
\end{array}\right]^{T}
\end{aligned}
$$

Apart from the linear EOM, a linear model of the MCA is required. While the $\boldsymbol{x}^{E O M}$ state vector is known from flight dynamics, the formulation of $\boldsymbol{x}^{M C A}$ depends on the used MCA. Equation 4 formulates a generic linear MCA structure.

$$
\begin{aligned}
\dot{\boldsymbol{x}}^{M C A} & =A^{M C A} \boldsymbol{x}^{M C A}+B^{M C A} \boldsymbol{u}^{M C A} \\
\boldsymbol{u}^{M C A} & =\left[\begin{array}{lll}
f_{y_{p}}^{E O M} & p^{E O M} & r^{E O M}
\end{array}\right]^{T}
\end{aligned}
$$

A system coupling, indicated by $\square^{C O U P}$, is designed to combine the systems in the form of Equations 6 to 8 .

$$
\begin{aligned}
& \dot{\boldsymbol{x}}^{E M D}=A^{E M D} \boldsymbol{x}^{E M D}+B^{E M D} \boldsymbol{u}^{E M D} \rightarrow\left[\begin{array}{c}
\dot{\boldsymbol{x}}^{\text {EOM }} \\
\dot{\boldsymbol{x}}^{M C A}
\end{array}\right]=\left[\begin{array}{cc}
A^{\text {EOM }} & 0 \\
A^{\text {COUP }} & A^{M C A}
\end{array}\right]\left[\begin{array}{l}
\boldsymbol{x}^{\text {EOM }} \\
\boldsymbol{x}^{M C A}
\end{array}\right]+\left[\begin{array}{c}
B^{\text {EOM }} \\
B^{\text {COUP }}
\end{array}\right] \boldsymbol{u}^{\text {EOM }} \\
& \boldsymbol{y}^{E M D}=C^{E M D} \boldsymbol{x}^{E M D}+D^{E M D} \boldsymbol{u}^{E M D} \rightarrow\left[\begin{array}{l}
\boldsymbol{y}^{\text {EOM }} \\
\boldsymbol{y}^{M C A}
\end{array}\right]=\left[\begin{array}{cc}
C^{\text {EOM }} & 0 \\
C^{\text {COUP }} & C^{M C A}
\end{array}\right]\left[\begin{array}{l}
\boldsymbol{x}^{\text {EOM }} \\
\boldsymbol{x}^{M C A}
\end{array}\right]+\left[\begin{array}{l}
D^{\text {EOM }} \\
D^{\text {COUP }}
\end{array}\right] \boldsymbol{u}^{\text {EOM }} \\
& \boldsymbol{y}^{E M D}=\left[\begin{array}{llllll}
f_{y_{p}}^{E O M} & p^{E O M} & r^{E O M} & f_{y_{p}}^{M C A} & p^{M C A} & r^{M C A}
\end{array}\right]^{T}
\end{aligned}
$$

The output vector $\boldsymbol{y}^{E M D}$ contains the motion cues as experienced by the pilot in the aircraft and in the simulator, indicated by $\square^{E O M}$ and $\square^{M C A}$, respectively. It must be noted that with this system description the motion cues for any trajectory can be compared. However, to reduce the dependency on specific trajectories, EMD only uses it to assess the inherent eigenmodes.

The combined linear system described by Equations 6 to 8 is used for the EMD analysis. First, eigenvectors of the state matrix $A^{E M D}$ are determined. To obtain eigenvectors expressed as pilot experienced quantities, the eigenvectors of the state matrix are multiplied with the output matrix $C^{E M D}$. With these eigenvectors, the imposed motion cue distortion can be determined by examining the difference between the eigenvector of the aircraft model and the coupled system, i.e. comparing the individual aircraft components of the eigenvector with their distorted simulator equivalents. 


\section{B. Linearized Flight Model}

This paper extends the earlier work on the EMD method [9] by applying it to an asymmetrical flight condition. Equation 9 shows the dimensional asymmetric EOM, which can be derived from flight dynamics. After some rewriting, these equations can be expressed in state-space form presented in Equations 1 to 3

$$
\begin{aligned}
W \cos \theta_{0} \phi+Y_{v} v+Y_{\dot{v}} \dot{v}+Y_{p} p+Y_{r} r+Y_{\delta_{a}} \delta_{a}+Y_{\delta_{r}} \delta_{r} & =m\left(\dot{v}+r V_{0}\right) \\
L_{v} v+L_{p} p+L_{r} r+L_{\delta_{a}} \delta_{a}+L_{\delta_{r}} \delta_{r} & =I_{x x} \dot{p}-I_{x z} \dot{r} \\
N_{v} v+N_{\dot{v}} \dot{v}+N_{p} p+N_{r} r+N_{\delta_{a}} \delta_{a}+N_{\delta_{r}} \delta_{r} & =I_{z z} \dot{r}-I_{x z} \dot{p} \\
\dot{\psi} & =\frac{r}{\cos \theta_{0}} \\
\dot{\phi} & =p+r \tan \theta_{0}
\end{aligned}
$$

Equation 9 is derived in the stability reference frame and assumes a steady, straight and symmetric flight condition with a flat non-rotating Earth. The CWA requires rotational rates and specific forces as input, which must therefore be the output of the EOM state-space system. The roll- and yaw rate are available as states, but an expression for sway specific force needs to be formulated.

Specific force is defined as the non-gravitational force per unit mass [1], which implies that the gravitational component needs to be removed from the lateral acceleration equation.

$$
\begin{aligned}
f_{y_{c g}}^{b} & =a_{y_{c g}}^{b}-g \cos \theta \sin \phi \\
& =\dot{v}+r u-p w-g \cos \theta \sin \phi
\end{aligned}
$$

The sway specific force, shown in Equation 10 , is defined in the body reference frame, as required for the CWA. As the EOM in Equation 9 are defined in the stability reference frame, a transformation from stability to body frame is required. It must be mentioned that this transformation is not required if the EOM are defined in body frame or the MCA uses the stability frame as a reference.

The next step is to express the sway specific force at the pilot station. This transformation becomes increasingly important for larger aircraft. For example, for an airliner, the distance between the centre of gravity and the pilot station is larger than for a small business jet. This increased distance acts as a moment arm, giving additional experienced specific forces due to rotational rates and accelerations. For small aircraft, the pilot is sitting close to the centre of gravity, which implies that this transformation is not very important. However, for the Cessna Citation 500 (the aircraft used in this paper) this distance is considered to be non-negligible. The transformation from centre of gravity to pilot station is given by Equation 11. For the Cessna Citation 500, the moment arms are $l_{x}=3.2[\mathrm{~m}]$ and $l_{z}=1.0[\mathrm{~m}]$, for horizontal and vertical distance, respectively [11].

$$
f_{y_{p}}=f_{y_{c g}}^{b}+\dot{p} l_{z}+\dot{r} l_{x}
$$

To construct the state-space output and feedthrough matrices, $C^{E O M}$ and $D^{E O M}$, respectively, linearization is required. Equation 12 shows the result of this linearization. Here, subscript 0 denotes the trim point of the linearization.

$$
f_{y_{p}}=\dot{v}-g \phi-u_{0} \alpha_{0} p+u_{0} r+l_{z} \dot{p}+l_{x} \dot{r}
$$

As a linear expression for sway specific force is derived, the $\boldsymbol{y}^{E O M}$ can be constructed. Roll- and yaw rates are directly available as states in the state-space, and the sway specific force can be constructed as a combination of states and inputs. This concludes the derivation of the state-space output and feedthrough matrices, where $\boldsymbol{y}^{E O M}$ is the resulting output vector, shown in Equations 7 and 8 .

\section{Linearized MCA}

The MCA yields positions and orientations to control the motion platform. To find the motion cue distortions caused by the MCA, the inputs and outputs need to be related to each other. Specific forces and rotational rates, defined in body reference frame, are inputs to the Classical Washout Algorithm (CWA), which is the MCA used in this study. The 
outputs are modified to output specific forces and rotational rates instead of positions and orientations, Figure 1 depicts this modification for the lateral CWA.

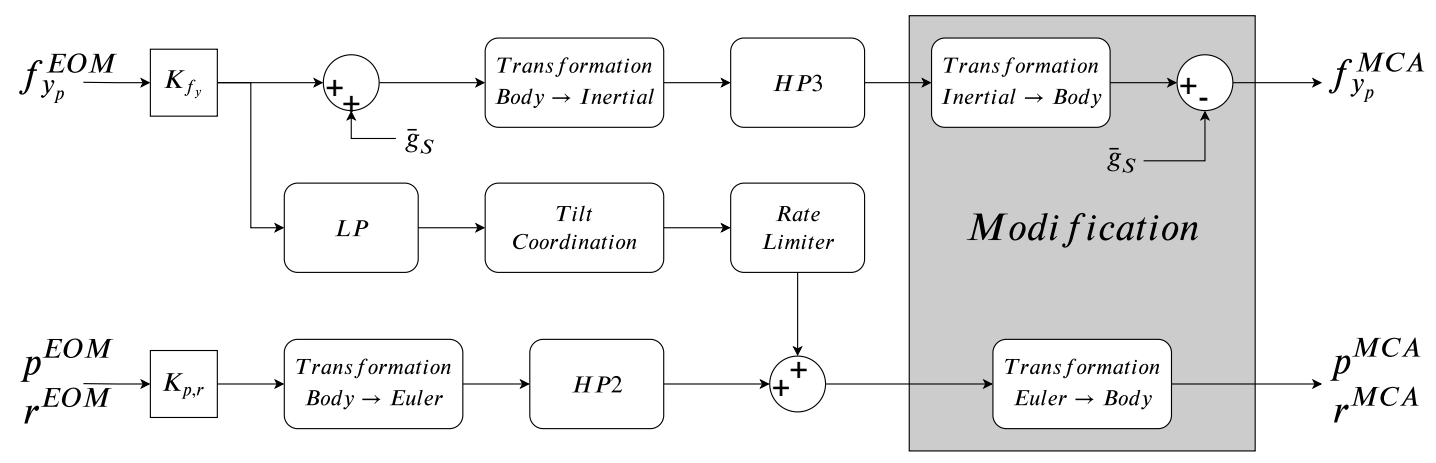

Fig. 1 The Classical Washout Algorithm Adapted for Lateral Motions [1]

The CWA is constructed for lateral motions only, so longitudinal channels are removed. As of nonlinearities, this CWA is linearized for the EMD analysis. Besides, a small angle approximation is used for the transformations, and the rate limiter in the tilt-coordination channel is removed. After linearization, this gives the state-space system- and input matrices, $A^{M C A}$ and $B^{M C A}$, respectively.

The state-space output matrix $\left(C^{M C A}\right)$ and feedthrough matrix $\left(D^{M C A}\right)$ are formulated to output sway specific force, roll rate, and yaw rate. The resulting output vector is $\boldsymbol{y}^{M C A}$, shown in Equations 7 and 8 .

\section{System Coupling}

As the next step in the EMD analysis, the linear models of the EOM and MCA are combined into a single state-space system. This is possible as the outputs of the EOM are inputs to the MCA (Figure 2). So-called coupling matrices are defined to express the MCA inputs as EOM states and inputs. For this, $B^{M C A}$ is reformulated as $A^{\text {COUP }}$ and $B^{C O U P}$, and $D^{M C A}$ is reformulated as $C^{C O U P}$ and $D^{C O U P}$. Finally, this gives a state-space system as in Equations 6 to 8 .

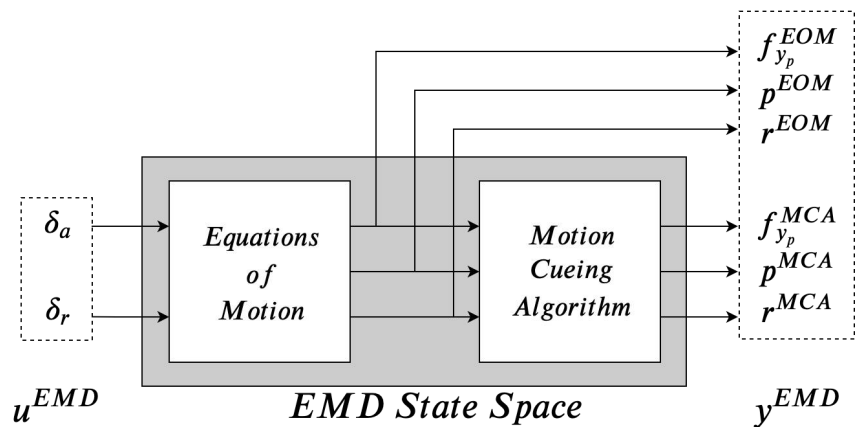

Fig. 2 EMD System Coupling

\section{E. Eigenvector Distortion Analysis}

When analyzing aircraft eigenmodes, eigenvalues are usually used, which provide information about the nature (e.g. periodic or aperiodic, stable or unstable), timing and decay of a mode. For example, the Dutch roll has two complex poles, meaning that it is a periodic mode, whereas the spiral mode has one real pole close to the imaginary axis, meaning that it is an aperiodic mode of slow nature.

$$
\begin{aligned}
& A^{E M D} \boldsymbol{x}_{i}^{E M D}=\lambda_{i}^{E M D} \boldsymbol{x}_{i}^{E M D} \\
& V_{\boldsymbol{x}}=\left[\begin{array}{lll}
\boldsymbol{x}_{1}^{E M D} & \cdots & \boldsymbol{x}_{n}^{E M D}
\end{array}\right]
\end{aligned}
$$


Additionally, eigenmodes can be analyzed using eigenvectors, which provide information on how the components of $\boldsymbol{x}^{E M D}$ interact in each eigenmode. Equation 13 presents the formulation for determining the $i^{t h}$ eigenvector and its associated eigenvalue for an individual mode. Eigenvectors of different modes can be combined in a matrix, $V_{\boldsymbol{x}}$, see Equation 14 These eigenvectors can be used to assess the MCA's motion cue distortions. First, eigenvectors of the $A^{E M D}$ matrix are determined, which are expressed in $\boldsymbol{x}^{E M D}$ states. To obtain the perceived motion cue distortions, these eigenvectors are multiplied with the output matrix, $C^{E M D}$, Equation 15 .

$$
V_{\boldsymbol{y}}=C^{E M D} V_{\boldsymbol{x}}
$$

The resulting eigenvectors, containing both undistorted aircraft and distorted simulator cues, are shown in Figure 3 for the lateral model of the Cessna Citation 500 in cruise configuration (Table 2) and representative MCA settings. In Figure 3 , the solid lines represent the motion cues as experienced by the pilot in the actual aircraft. Furthermore, the dashed lines represent the motion cues as experienced in the flight simulator. The magnitude and phase distortions between the solid and dashed lines are directly caused by the MCA and form the basis of the EMD method.

It must be stated that a comparison in magnitudes between specific forces and rotational rates has to take into account their different units. For example, in Figure 3 the magnitude of the sway specific force components is larger than that of the roll rate. However, it would be wrong to conclude that the sway specific force is thus a more dominant motion cue in this mode. Even though the linear system is on first sight dimensionless, the relative units between the individual states are still present in the state space representation. This issue of dimensionality is the motivation for the EMD method extension described in Section

The magnitude distortion is defined as the ratio between the magnitudes of $\square^{E O M}$ and $\square^{M C A}$ components. Additionally, phase distortion is defined as the angle between these components in the complex plane. If a complex eigenvector that corresponds to the positive conjugate eigenvalue is visualized, counter-clockwise and clockwise phase distortion indicates phase lead and phase lag between the two components with respect to the actual aircraft, respectively.

Figure 3a depicts eigenvectors corresponding to the Dutch roll for an example MCA parameter set. The following observations can be made; sway specific force, $f_{y_{p}}^{M C A}$, has a magnitude distortion of $\left|f_{y}\right|=\frac{\left|f_{p}^{M C A}\right|}{\left|f_{y p}^{E O M}\right|}=0.64$ and a phase distortion of $\angle f_{y}=16.3^{\circ}$ lead (counter-clockwise) for this tuning. Furthermore, the roll rate, $p^{M C A}$, has a magnitude distortion of $|p|=0.62$ and phase distortion of $\angle p=18.1^{\circ}$ lead. Finally, the yaw rate, $r^{M C A}$, has a magnitude distortion of $|r|=0.69$ and phase distortion of $\angle r=24.5^{\circ}$ lead. These observations are supported by analyzing the time domain response. Figure 4 illustrates the time-traces to a zero-input response, in which $2 \operatorname{Re}\left(v_{i}\right)$ is the initial condition. For this initial condition, $v_{i}$ is one of the two (complex) eigenvectors associated with the (complex) eigenmode. This means that both the Dutch roll eigenvectors (which are complex conjugates) are used to excite the Dutch roll eigenmode, and their (complex) responses are effectively combined to yield a real system response.

The EMD distortions can be compared to the distortions found by OMCT at the frequency of the Dutch roll eigenmode. The natural frequency of the Dutch roll is $3.2738 \mathrm{rad} / \mathrm{s}$. The nearest OMCT test frequency is $3.9810 \mathrm{rad} / \mathrm{s}$ and gives as distortions; for sway specific force (test 8$)\left|f_{y}\right|=0.75$ and $\angle f_{y}=29.7^{\circ}$ lead, for roll rate (test 3$)|p|=0.68$ and $\angle p=19.9^{\circ}$ lead and for raw rate (test 5) $|r|=0.68$ and $\angle r=19.9^{\circ}$ lead. Especially the coupled sway and roll channels show relatively large differences between EMD and OMCT results, illustrating the differences between using eigenmodes and isolated sinusoids.

Magnitude distortions for the other eigenmodes, using EMD, are shown in Table 1 As the aperiodic roll and spiral mode are both aperiodic (real) modes, only a magnitude distortion and possibly a sign change can be obtained. Interesting to

Table 1 Example Magnitude- and Phase Distortions

\begin{tabular}{cc|l|l}
\hline \hline \multicolumn{2}{c|}{ Dutch Roll } & Aperiodic Roll & \multicolumn{1}{l}{ Spiral Mode } \\
\hline$\left|f_{y}\right|=0.64$ & $\angle f_{y}=16.3^{\circ}$ lead & $\left|f_{y}\right|=1.59$ & $\left|f_{y}\right|=0.80$ \\
$|p|=0.62$ & $\angle p=18.1^{\circ}$ lead & $|p|=0.76$ & $|p|=0.003$ \\
$|r|=0.69$ & $\angle r=24.5^{\circ}$ lead & $|r|=1.02$ & $|r|=0.00$ \\
\hline \hline
\end{tabular}




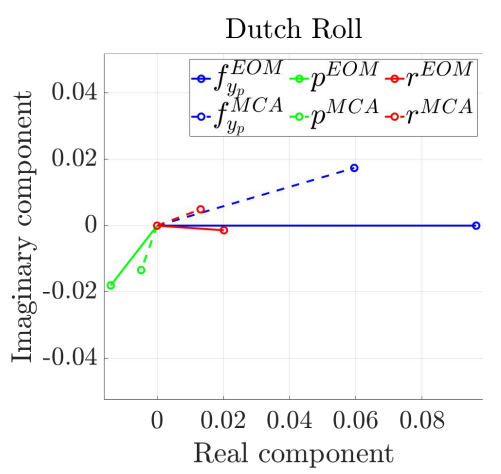

(a) Dutch Roll

$\left(\lambda_{d r}=-0.2778 \pm 3.2616 i\right)$

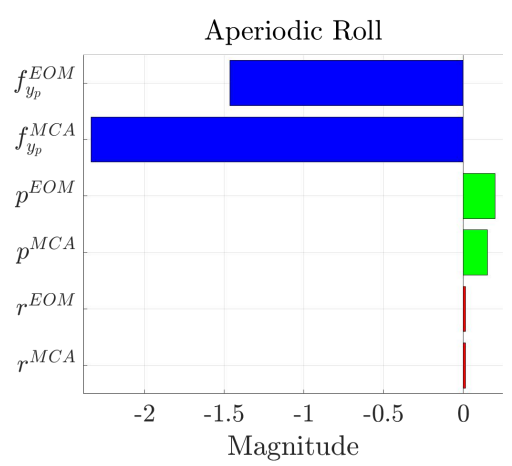

(b) Aperiodic Roll

$\left(\lambda_{\text {ar }}=-4.0740\right)$

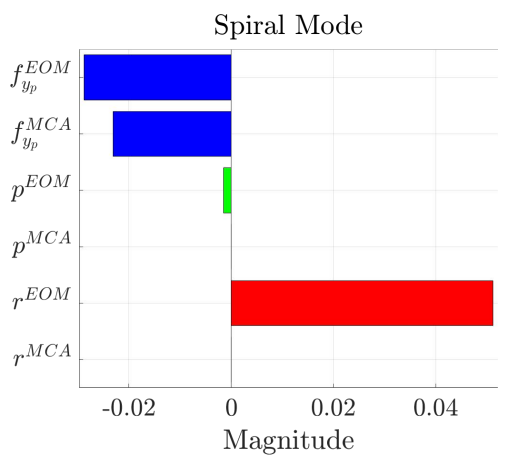

(c) Spiral Mode

$\left(\lambda_{s m}=-0.0018\right)$

Fig. 3 Example of Eigenvector Distortion Plots
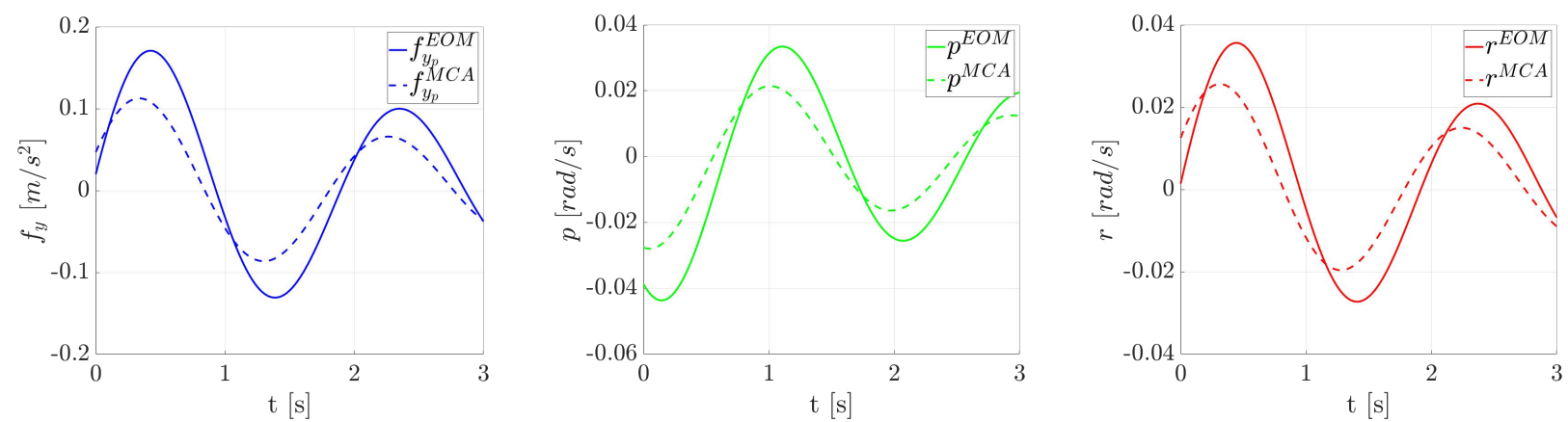

Fig. 4 Time Histories of Dutch Roll Eigenmode Excitation
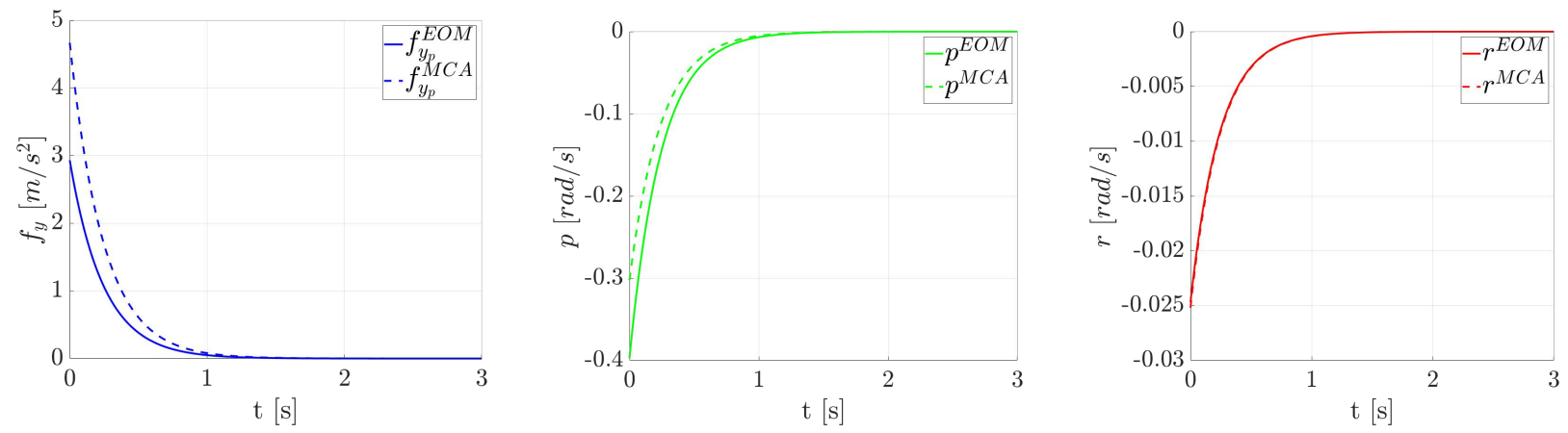

Fig. 5 Time Histories of Aperiodic Roll Eigenmode Excitation
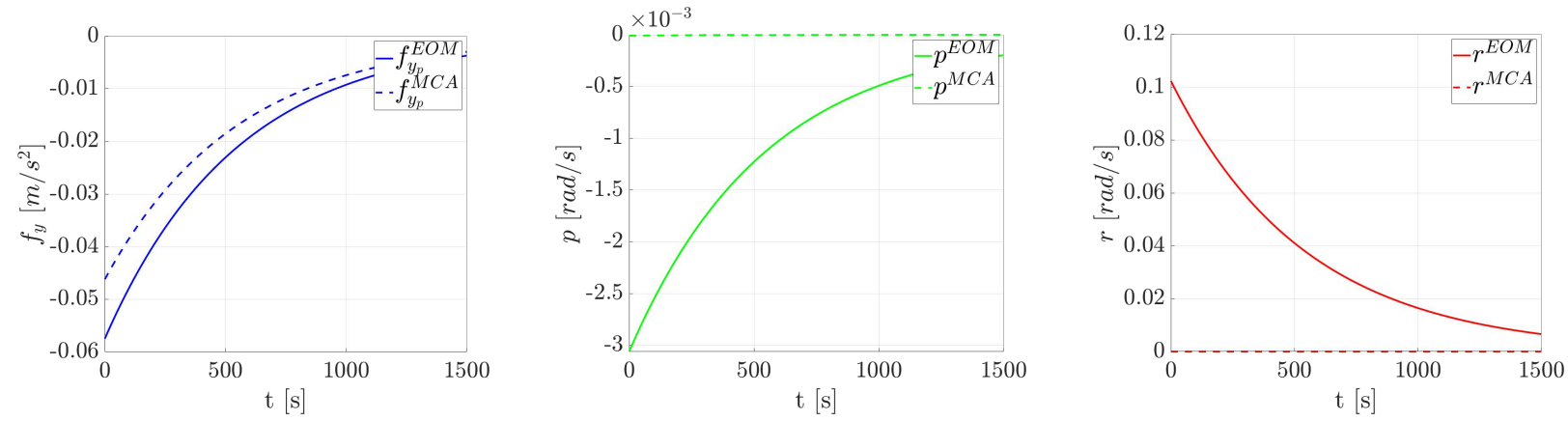

Fig. 6 Time Histories of Aperiodic Spiral Mode Excitation 
observe is the large magnitude distortion of the roll and yaw rate for the spiral mode. The time domain representation of the spiral mode (Figure 6 depicts these motion cues as essentially zero. Which is explained by the eigenvalue being close to the imaginary axis, e.g., $\lambda_{s m}=-0.0018$, and the high-pass rotation channels strongly attenuating these cues.

All linear aircraft responses can be described in the time domain by a continuously varying combination of eigenmodes. The influence of a particular mode at a particular time can be expressed by the system's modal response. The modal coordinate transformation, which can be computed as $z(t)=V_{x}^{-1} x^{E M D}(t)$, yields (complex) variables representing the system's modal response in the time domain. The Mode Participation Factor (MPF) is the absolute value of this complex variable $z$ and reveals how each mode participates at each time point in a particular manoeuvre [12]. The MPF also depends on pilot inputs, i.e. if the pilot is exciting a particular mode. Moreover, MPFs have been used to identify dominant modes in a particular manoeuvre or task [8].

MPFs are useful to identify which modes are excited in a particular manoeuvre. In the experiment performed by Stoev et al. [9], the short period eigenmode was excited by the pilot as illustrated by the dominant MPF. The short period is a desired mode necessary for performing manoeuvres, such as pitch captures, and is easily excited. In that case, the mode's MPF hinted at its importance during the maneuver. On the other hand, the Dutch roll is an unpleasant mode and usually damped with a yaw damper. If the aircraft lacks a yaw damper, the pilot has to suppress this mode themselves, possibly aided by the aircraft's or simulator's motion cues. If the pilot is successful, the Dutch roll's MPF would become quite small, belying the mode's importance in the pilot's control behaviour. As this study focuses on the lateral flight dynamics, and the Dutch roll in particular, it differs in its use of the MPF from the earlier study performed by Stoev et al.

Table 2 Asymmetric Stability and Control Derivatives for Cessna Citation 500, condition: Cruise

\begin{tabular}{rrllllll}
\hline \hline$C_{Y_{\beta}}=-0.0750$ & $C_{l_{\beta}}=-0.1061$ & $C_{n_{\beta}}=0.1305$ & $V=160.3[\mathrm{~m} / \mathrm{s}]$ & $C_{L}=0.2239[-]$ \\
$C_{Y_{p}}=-0.0109$ & $C_{l_{p}}=-0.5209$ & $C_{n_{p}}=-0.0672$ & $h=5000[\mathrm{~m}]$ & $\rho$ & $\rho$ & $\rho$ & $0.7361\left[\mathrm{~kg} / \mathrm{m}^{3}\right]$ \\
$C_{Y_{r}}=$ & 0.8499 & $C_{l_{r}}=-0.1522$ & $C_{n_{r}}=-0.2089$ & $S=24.2\left[\mathrm{~m}^{2}\right]$ & $K_{X X}^{2}=0.019[-]$ \\
$C_{Y_{\delta_{a}}}=-0.0400$ & $C_{l_{\delta_{a}}}=-0.1848$ & $C_{n_{\delta_{a}}}=-0.0140$ & $m=5207[\mathrm{~kg}]$ & $K_{Z Z}^{2}=0.042[-]$ \\
$C_{Y_{\delta_{r}}}=0.2300$ & $C_{l_{\delta_{r}}}=0.0358$ & $C_{n_{\delta_{r}}}=-0.0947$ & $b=13.36[\mathrm{~m}]$ & $K_{X Z}=0.002[-]$ \\
\hline \hline
\end{tabular}

\section{Perceptual Eigenmode Distortion}

\section{A. Methodology}

The EMD method was introduced to overcome some of the shortcomings of the OMCT method. Instead of using an isolated frequency response at discrete frequencies, a different method is used to perform the distortion analysis, in which motion cue distortions are investigated using eigenvectors. Yet the method as used until now has a limitation, in that the resulting components of the eigenvectors for specific forces and rotational rates cannot easily be compared to one-another because of their different units. This implies that a dominant motion cue is not readily apparent by comparing the component magnitudes.

The Perceptual Eigenmode Distortion (PEMD) introduced in this paper extends the EMD method with a perception model, which directly addresses the component magnitude mismatch present in EMD, and aims to include characteristics of the pilot's perceptual system in the analysis. Initially, the PEMD had two additions. The EMD-derived eigenvectors assume a vestibular system that perceives motion cues directly, whereas an actual vestibular system introduces a additional magnitude and phase distortions. The first proposed addition was to include a linear model of the vestibular system. However, as the EOM outputs, $\boldsymbol{y}^{E O M}$, and MCA outputs, $\boldsymbol{y}^{M C A}$, pass through the same (model of the) vestibular system, their relative magnitude and phase distortions remain the same, limiting the usefulness of this addition.

Also, in the derivation, this addition increased the method's complexity as the number of states almost doubled. Another point of criticism is that the vestibular system model is an idealization and simplification of an actual vestibular system. Given the resulting limited added value of this addition, the linear vestibular system addition is ultimately left out of the proposed PEMD method.

The second proposed addition is to re-express the output vector in terms of motion perception thresholds instead of engineering units. The threshold for motion perception is the value that motion is not perceived by humans anymore 
[13]. After re-expressing the output vector, all motion cues have the same unit, which can be called the threshold unit and is fully dimensionless. When motion cues in an eigenmode have the same magnitude in threshold units, these cues have the same probability of exceeding its threshold when exciting that mode with a random magnitude. As the output vector is re-expressed, the output matrix is also changed $\left(C^{E M D} \rightarrow C^{P E M D}\right)$. The same eigenvector analysis can now be performed, using the new output matrix, which results in eigenvectors all expressed in threshold units. As the eigenvectors now have the same units for all of their components, these components of the eigenvectors can be compared to one-another and the dominance of individual motion cues within an eigenmode can be identified.

\section{B. Perception Model Extension}

A problem with using perception thresholds is that these vary per person and depend on factors such as workload and task [2]. Furthermore, these thresholds tend to vary per frequency [14] and are affected by motions in different directions. Even though this paper attempts to incorporate perception thresholds in the eigenvector analysis, developing a complete threshold model would be too ambitious as this stage. Therefore, the perception thresholds used are chosen as constants.

The threshold values used are obtained from a recent simulator study [13]. As those values are not constant with frequency, a simplification was introduced by using average values over the frequency range. [14]. The used threshold values for sway specific force, roll- and yaw rate are, $\breve{f_{y}}=0.0743\left[\mathrm{~m} / \mathrm{s}^{2}\right], \check{p}=0.00521[\mathrm{rad} / \mathrm{s}]$ and $\check{r}=0.0166[\mathrm{rad} / \mathrm{s}]$, respectively.

The reformulated output vector, Equation 17 is obtained by dividing the elements of the $C^{E M D}$ matrix by its associated threshold value, which is illustrated in Equation 16 . In here, the symbol $\check{a}$ indicates that threshold units are used. As all outputs are now scaled to the same (threshold) unit, the magnitudes of the different output states can directly be compared.

$$
\begin{aligned}
& C^{P E M D}=\left[\begin{array}{l}
C^{E M D}(1) / \check{f} \\
C^{E M D}(2) / \check{p} \\
C^{E M D}(3) / \check{r} \\
C^{E M D}(4) / \check{f} \\
C_{y}^{E M D}(5) / \check{p} \\
C^{E M D}(6) / \check{r}
\end{array}\right] \\
& \check{\boldsymbol{y}}^{\text {PEMD }}=\left[\begin{array}{llllll}
\check{f}_{y_{p}}^{E O M} & \check{p}^{E O M} & \check{r}^{E O M} & \check{f}_{y_{p}}^{M C A} & \check{p}^{M C A} & \check{r}^{M C A}
\end{array}\right]^{T}
\end{aligned}
$$

\section{Eigenvector Distortion Analysis}

Now, the eigenvector analysis is performed for PEMD in the same way as for EMD. First, the eigenvectors of the $A^{E M D}$ matrix are determined, which are expressed in $\boldsymbol{x}^{E M D}$ states. To obtain the motion cue distortions expressed in threshold units, the eigenvectors are multiplied with the new output matrix, $C^{P E M D}$ :

$$
V_{\check{y}}=C^{P E M D} V_{\boldsymbol{x}}
$$

These new eigenvectors are used to determine the motion cue distortions. The individual magnitude- and phase distortions are identical to the EMD's, as each relative distortion remains unchanged. Figure 7 shows the new eigenvectors.

As all components of the eigenvectors are now expressed in the same unit, the magnitudes can be compared to one-another. Therefore the following observations can be made; for the Dutch roll, Figure $7 \mathrm{a}$, roll rate is now shown to be the dominant motion cue. Also, sway specific force and yaw rate are about equally perceivable within the mode. For the aperiodic roll, Figure $7 \mathrm{~b}$, roll rate is the dominant motion cue and yaw rate is the least dominant. For the spiral mode, Figure $7 \mathrm{c}$, yaw rate is the most dominant motion cue. Yet, this motion cue is fully attenuated by the MCA, as evident from the zero magnitude of $\check{r}^{M C A}$. Similarly, the simulated roll rate is negligible.

It must be noted that within a certain maneuver, the magnitudes of the eigenvectors in Figure 7 vary in time, e.g. the magnitudes gets smaller as an undisturbed mode decays. Therefore, the axes contain no numbers. However, the relative magnitudes and phase remain always the same over time for a given mode and flight condition. 


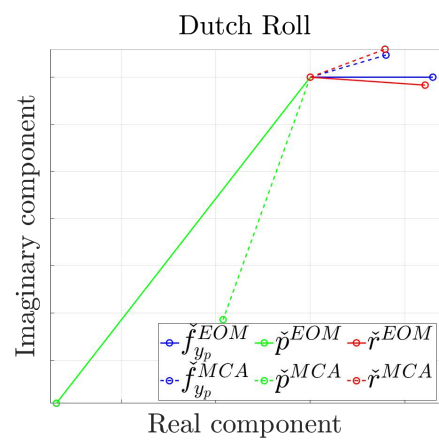

(a) Dutch Roll

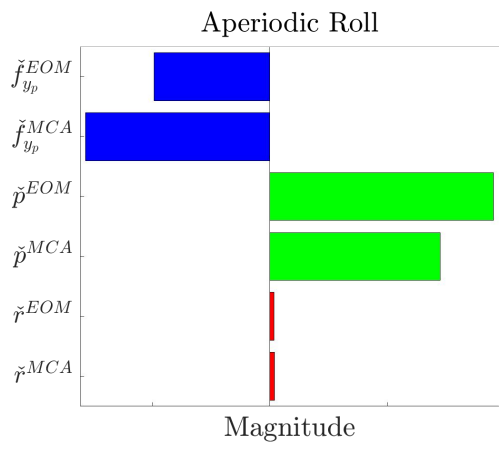

(b) Aperiodic Roll

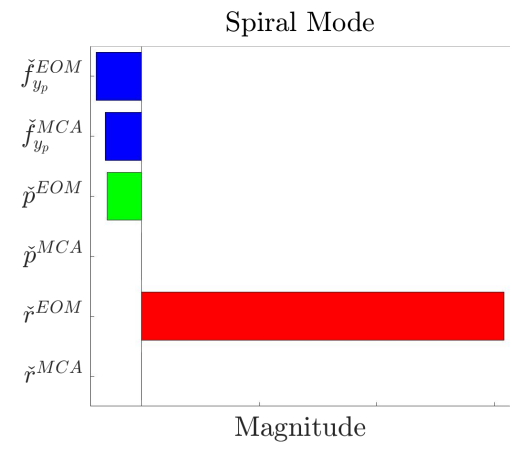

(c) Spiral Mode

Fig. 7 Example of Eigenvector Distortion Plots

It is hypothesized that a larger eigenvector component magnitude implies a larger contribution of a motion cue in an eigenmode. It must be stated that this contribution depends on the flight condition. In this paper, the Cessna Citation 500 in cruise condition is used, as defined in Table 2. The contribution of each motion cue per eigenmode for a given flight condition, which is defined as e.g., $W_{\square}=\left|\check{\check{a}}^{E O M}\right| /\left(\left|\breve{f}_{y_{p}}^{E O M}\right|+\left|\check{p}^{E O M}\right|+\left|\check{r}^{E O M}\right|\right)$ for a given eigenmode, is given in Table 3 This contribution can be used as a weighting factor for tuning, as described in next section.

Table 3 Eigenmode Motion Cue Contribution for Flight Condition Table 2

\begin{tabular}{l|ccc}
\hline \hline & $f_{y}$ & $p$ & $r$ \\
\hline Dutch Roll & $W_{f_{y}}^{D R}=0.188$ & $W_{p}^{D R}=0.635$ & $W_{r}^{D R}=0.177$ \\
Aperiodic Roll & $W_{f_{y}}^{A R}=0.337$ & $W_{p}^{A R}=0.651$ & $W_{r}^{A R}=0.013$ \\
Spiral Mode & $W_{f_{y}}^{S M}=0.103$ & $W_{p}^{S M}=0.078$ & $W_{r}^{S M}=0.819$ \\
\hline \hline
\end{tabular}

\section{Tuning Algorithm}

In the latest OMCT evaluation method, magnitude and phase distortions are evaluated separately. The study done by De Ridder and Roza [15] uses OMCT for automatically tuning the MCA parameters. They used an optimization criterion that combined the phase and magnitude distortions with somewhat arbitrary weighing factors. To address the relative weighing problem, the current study takes inspiration from earlier work from the control loading domain.

In a study done by $\mathrm{Fu}$ [16] it was found that a change of dynamics in control loading is not perceived by humans when the change in eigenvalue that occurs fall within a threshold region in the complex plane. For control loading, this threshold region seems to be a square. As a result, it does not matter whether it is a magnitude distortion and/or phase distortion. As long as the distortion is within this threshold region, it is not perceived by the human operator. Although Fu considered the system's eigenvalue and PEMD is focused on the system's eigenvector, the similarity inspired a new way of defining an objective function for tuning.

For PEMD, rather than optimizing only the magnitude distortion or phase distortion, an objective function is formulated in which the Euclidean distance between the $\square^{E O M}$ and $\square^{M C A}$ components is minimized. Figure 8 illustrates this Euclidean distance, $D_{\square}^{\square}$ with the top index denoting the mode and the bottom index the cue. Equation 19 shows the Euclidean distance for sway specific force of the Dutch roll. The $\boldsymbol{p}$ indicates the MCA parameters, which can be varied to change this distance. The other distances are formulated similarly.

$$
D_{f_{y}}^{D R}(\boldsymbol{p})=\left\|\check{f}_{y_{p}}^{E O M}-\check{f}_{y_{p}}^{M C A}(\boldsymbol{p})\right\|_{D R}
$$

Next, the contributions of each motion cue per eigenmode, from Table 3 is added. For example, in Figure 8a the magnitude of roll rate, $\breve{p}^{E O M}$, is larger than that of the sway specific force, $\breve{f}_{y_{p}}^{E O M}$, and yaw rate, $\check{r}^{E O M}$. Consequently, it is more important to minimize the Euclidean distance for roll rate than for sway specific force and yaw rate. By using 
a cue's individual contribution to a mode as a weighing factor $W_{\square}^{\square}$ on its associated Euclidean distance, an optimization cost function can be defined. Equations 20 to 22 show the cost functions, $C^{\square}$, for Dutch roll, aperiodic roll and spiral mode, respectively.

$$
\begin{aligned}
& C^{D R}(\boldsymbol{p})=W_{f_{y}}^{D R} \cdot D_{f_{y}}^{D R}(\boldsymbol{p})+W_{p}^{D R} \cdot D_{p}^{D R}(\boldsymbol{p})+W_{r}^{D R} \cdot D_{r}^{D R}(\boldsymbol{p}) \\
& C^{A R}(\boldsymbol{p})=W_{f_{y}}^{A R} \cdot D_{f_{y}}^{A R}(\boldsymbol{p})+W_{p}^{A R} \cdot D_{p}^{A R}(\boldsymbol{p})+W_{r}^{A R} \cdot D_{r}^{A R}(\boldsymbol{p}) \\
& C^{S M}(\boldsymbol{p})=W_{f_{y}}^{S M} \cdot D_{f_{y}}^{S M}(\boldsymbol{p})+W_{p}^{S M} \cdot D_{p}^{S M}(\boldsymbol{p})+W_{r}^{S M} \cdot D_{r}^{S M}(\boldsymbol{p})
\end{aligned}
$$

Finally, an overall objective function can be formulated. The objective function, as in Equation 23, minimizes the Euclidean distance for the eigenvectors in the selected eigenmodes. Eigenmodes can be prioritized by changing the values of $\alpha, \beta$ and $\gamma$, where $\alpha+\beta+\gamma=1$ and $0 \leq \alpha, \beta, \gamma \leq 1$.

$$
\min : \quad Z(\boldsymbol{p})=\alpha \cdot C^{D R}(\boldsymbol{p})+\beta \cdot C^{A R}(\boldsymbol{p})+\gamma \cdot C^{S M}(\boldsymbol{p})
$$

Ideally, all Euclidean distances would be zero. However, most flight simulators have motion limitations. The motion is limited by the actuators driving the motion platform. Apart from the actuator lengths, the maximum velocity and acceleration it can achieve are limited. Therefore, actuator limitations are added to the constrained optimization algorithm. Equations 24 to 26 show the actuator constraints in length, velocity and acceleration, respectively.

$$
\begin{aligned}
\boldsymbol{l}_{\text {min }}+B_{l} \leq & \boldsymbol{l}_{\text {response }}(\boldsymbol{p}, \boldsymbol{m}) \leq \boldsymbol{l}_{\text {max }}-B_{u} \\
& \left|\boldsymbol{v}_{\text {response }}(\boldsymbol{p}, \boldsymbol{m})\right|_{\text {max }} \leq \boldsymbol{v}_{\max } \\
& \left|\boldsymbol{a}_{\text {response }}(\boldsymbol{p}, \boldsymbol{m})\right|_{\max } \leq \boldsymbol{a}_{\text {max }}
\end{aligned}
$$

In here, $\boldsymbol{m}$ represents a representative manoeuvre, which can be used to ensure that the available motion space is not exceeded. Buffers can be introduced to further restrict the available motion space, i.e., the lower- and upper buffer distances ( $B_{l}$ and $B_{u}$, respectively) can be increased.

The formulation of the tuning algorithm allows for some flexibility. The user can specify which eigenmode is tuned. Furthermore, different representative manoeuvres can be used to optimize for different training tasks. Also, the actuator buffer distance in Equation 24 can be used to limit the motion space. The tuning algorithm can also be extended to include symmetric flight.

One approach for the actual optimization is to use genetic algorithms. Genetic algorithms are stochastic global search and optimization methods which apply the principle of natural biological evolution through survival of the fittest [17]. In this study, the automatic tuning algorithm was implemented in Matlab/Simulink using the built-in genetic algorithm, i.e. the ga function.

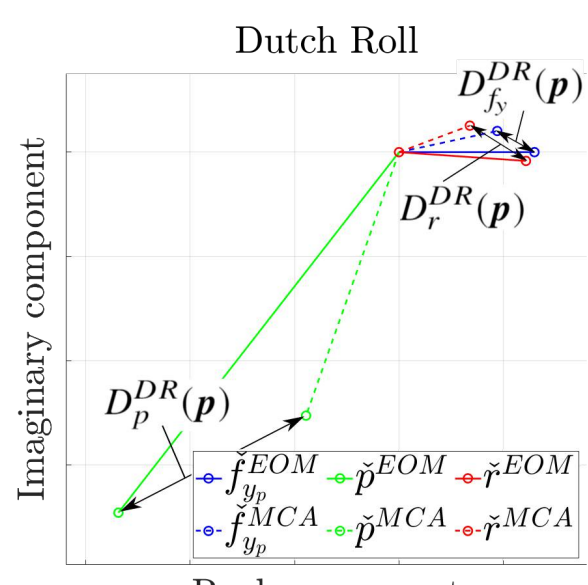

Real component

(a)

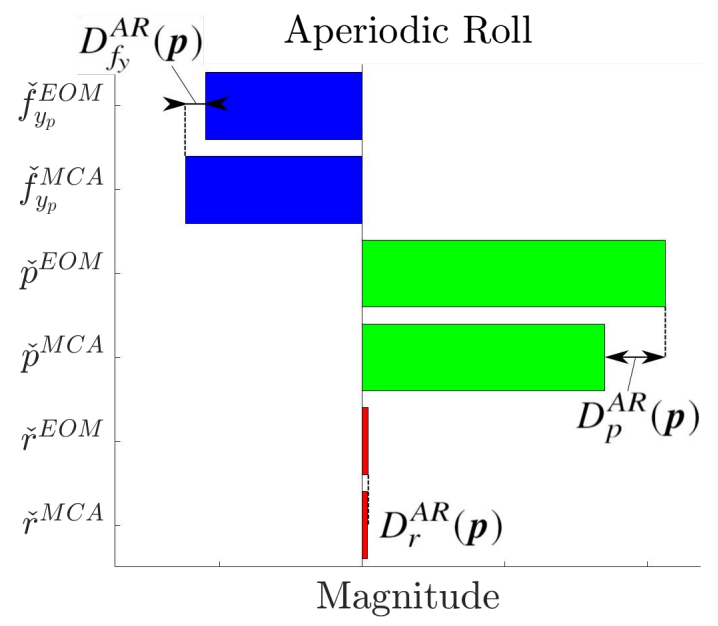

(b)

Fig. 8 Euclidean Distance 


\section{Pilot-in-the-Loop Experiment}

To investigate the potential of the PEMD method, the automatic tuning algorithm, and to validate the resulting motion cueing solution, a pilot-in-the-loop experiment was performed with the TU Delft's SIMONA Research Simulator [10]. The simulated aircraft was a nonlinear model of the Cessna Citation 500 business jet. Three different MCA configurations were used in the experiment, which were subjectively compared in a double-blind pairwise pattern and with an objective performance metric. In total seven pilots participated in the experiment. After a discussion of the three MCA configurations, some general information of the participants is given, which is followed by a review of the apparatus and experiment task. Finally, hypotheses and corresponding dependent measures are given.

\section{A. Independent Variables: MCA Configurations}

This study applied the tuning algorithm to a lateral model of the Cessna Citation 500 in cruise configuration, Table 2 The lateral model has three eigenmodes, namely: Dutch roll, aperiodic roll and spiral mode. Ideally, the MCA parameters are optimized for all eigenmodes concurrently. Yet, only the Dutch roll and aperiodic roll were selected for tuning the configurations. The spiral mode was left out as motion simulators have a limited motion space and are thus better in simulating high-frequency motions than low-frequency [1]. Furthermore, the Dutch roll and aperiodic roll can be excited with a small deviation from the trim conditions, whereas large deviations are required for the spiral mode.

This paper analyzed a total of three CWA configurations. The first configuration is the baseline configuration, $B$, which was separately tuned by an MCA tuning expert and was based on experience and rules of thumb. Additionally, this configuration satisfied the latest OMCT criteria, specified in ICAO - Manual of Criteria for the Qualification of Flight Simulation Training Devices [6]. The second configuration, $O$, was tuned to only satisfy the OMCT tests. The last configuration, $P$, was tuned according to the tuning algorithm of Section IV] For this, the spiral mode was excluded. Pilot feedback from previous experiments indicated that simultaneous tuning could improve the realism of motion cues [9], and therefore $\alpha=\beta=0.5$ was chosen. For all configurations, a representative Dutch roll manoeuvre, in which the Dutch roll was excited by the pilot with a repeated rudder input, was simulated to ensure that the found MCA settings did not exceed the available motion space. It is important to note that $\boldsymbol{m}$, in Equations 24 to 26, represent the time history of this representative manoeuvre. Tables 4 and 5 present the resulting CWA parameter settings and distortions, respectively. As the yaw cue was the least dominant motion cue for both the Dutch roll and aperiodic roll, all configurations were designed to have identical MCA parameters for yaw. These yaw parameters were tuned to satisfy the OMCT yaw due to yaw test, i.e. test 5 .

For all configurations, the eigenvector distortions are depicted in Figures 9 to 11 and quantified in Table 5 . From inspection, it can be observed that configurations $B$ and $O$ have rather similar distortions. For the Dutch roll, both configurations preserve a rather low phase distortion for $f_{y}$ and $p$. For aperiodic roll, both configurations exhibit reasonable magnitude distortions of no more than $17 \%$. However, both contain a sign change for the roll cue, i.e., the roll cue is simulated in the opposite direction in this mode. Configuration $P$ has the lowest phase distortion in $f_{y}$ for the Dutch roll. The $p$ has a larger phase distortion but almost no magnitude distortion, making the Euclidean distance smaller than for the $B$ - and $O$ configuration. From Figure $11 \mathrm{~b}$ it can be observed that the $f_{y}$ and $p$ motion cues for the aperiodic roll exactly match the aircraft dynamics.

The eigenvalues for Dutch roll, aperiodic roll and spiral mode, shown in Equation 27, aid in the understanding of how the tuning algorithm behaves. These eigenvalues provide information about the nature, timing and decay of an eigenmode. As the Dutch roll eigenvalue is closer to the imaginary axis than the eigenvalue of the aperiodic roll, the decay is slower. To cue the Dutch roll more realistically, the motion platform would require more motion space. It must be stated that the motion space, in Equations 24 to 26 are configured such that the available motion space is comparable to the used motion space of configurations $B$ and $O$. Otherwise, pilots can identify the $P$ configuration by only focusing on the magnitude of the motion. Furthermore, would the pilot make a control error, the simulator would automatically run to its limits if the $P$ configuration was tuned to use all available motion space.

$$
\lambda_{d r}=-0.2778 \pm 3.2616 i \quad \lambda_{a r}=-4.0740 \quad \lambda_{s m}=-0.0018
$$

Offline OMCT tests were applied to the three MCA configurations. In here, motion platform dynamics were excluded [18]. The results of these tests are depicted in Figure 12. The configurations $B$ and $O$ complied with the fidelity boundaries defined by ICAO 9625 [6]. As this is an offline test, it must be noted that for example, the infractions in Figure 12c will likely be within the boundaries due to an additional lag of the actuator dynamics [18]. 
Dutch Roll

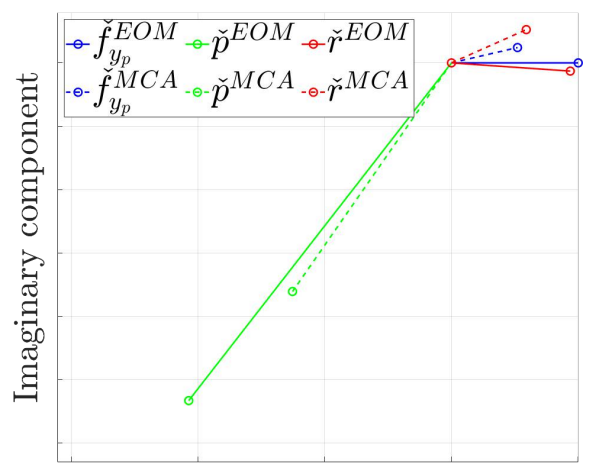

Real component

(a)

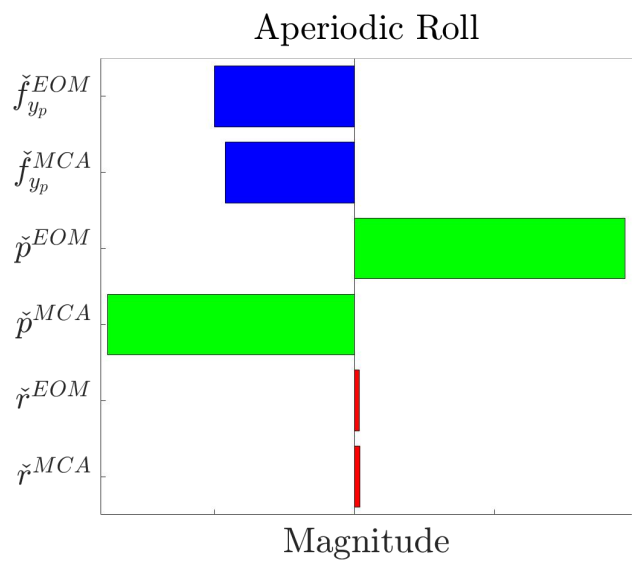

(b)

Fig. 9 Configuration $B$

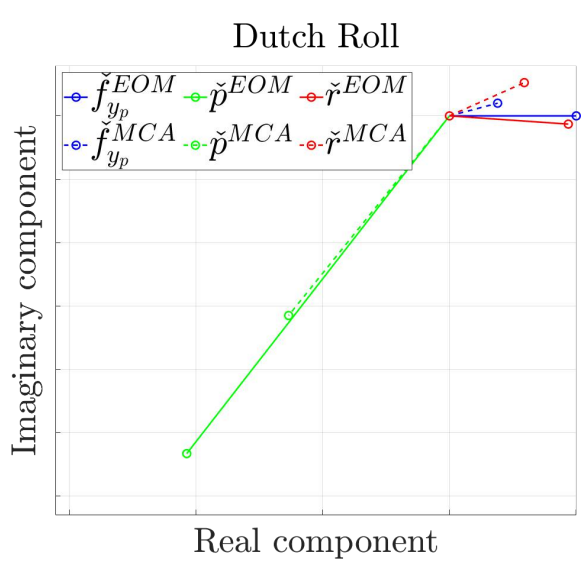

(a)

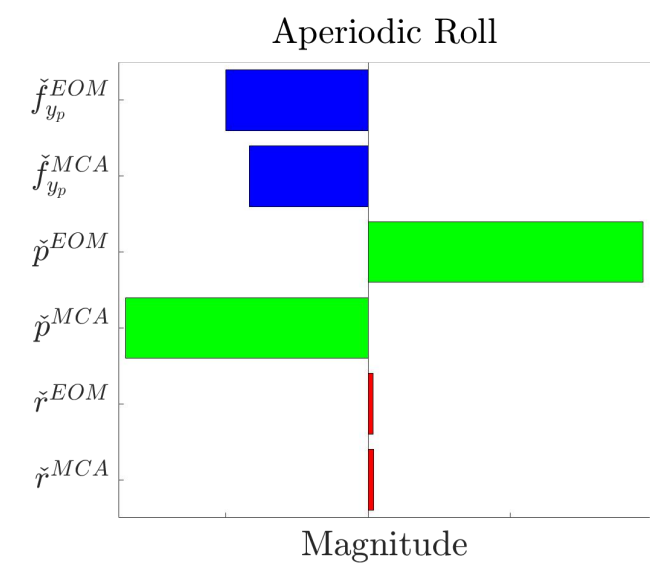

(b)

Fig. 10 Configuration $O$

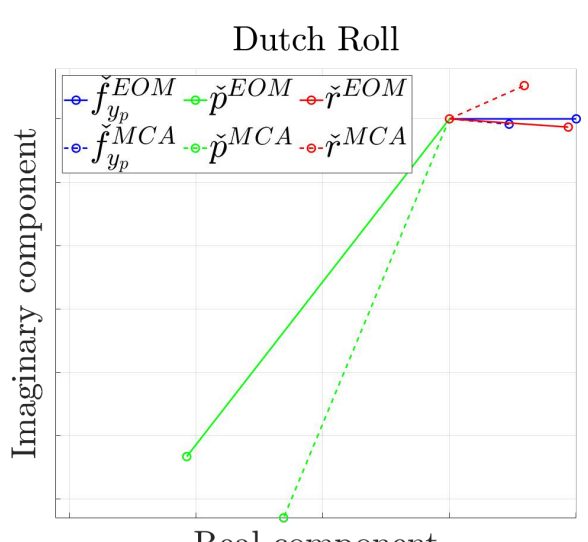

Real component

(a)

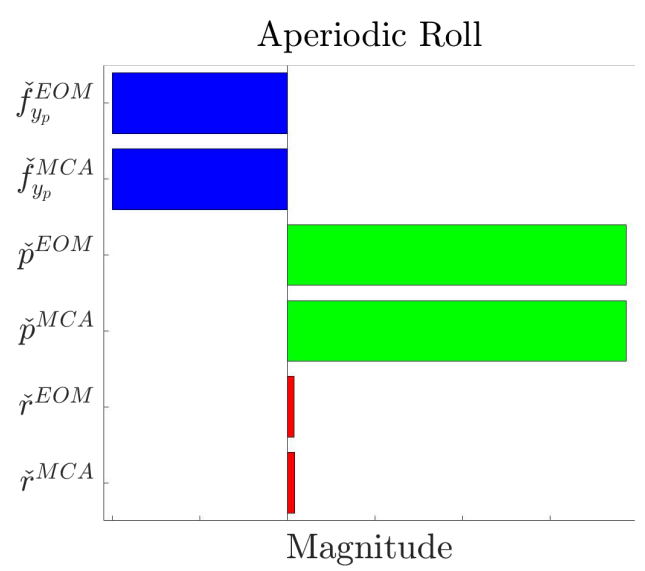

(b)

Fig. 11 Configuration $P$ 
Roll due to Roll
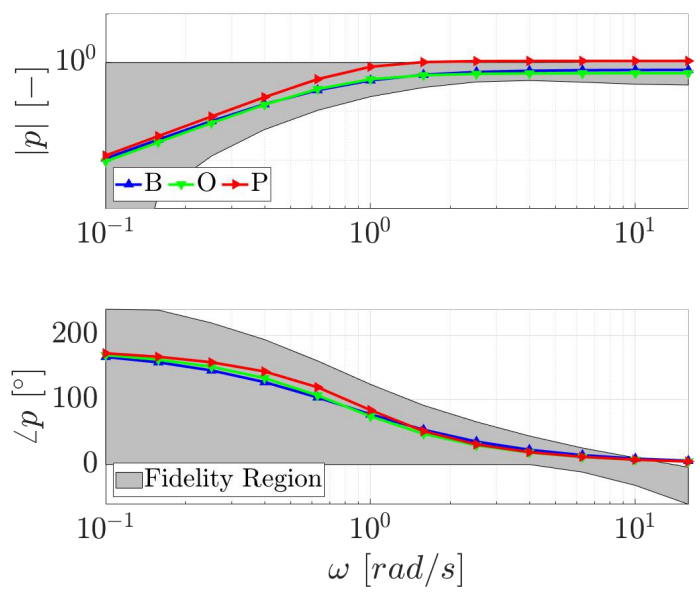

(a) Test 3
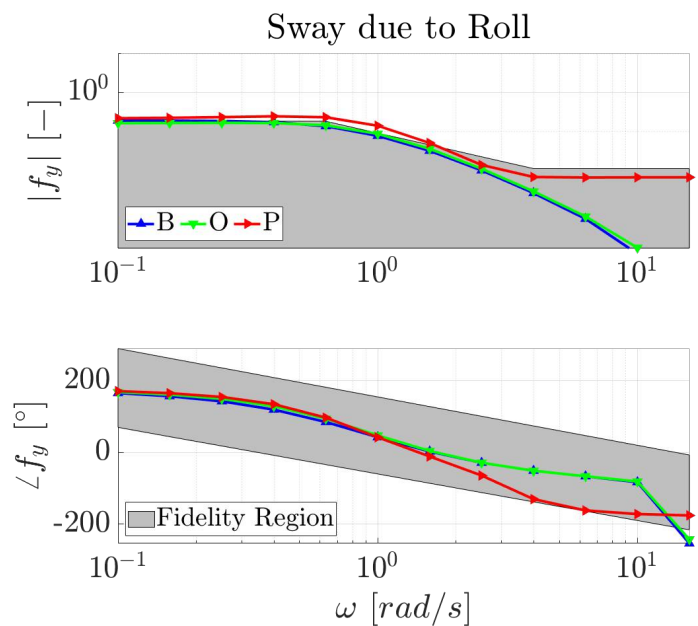

(b) Test 4

Yaw due to Yaw
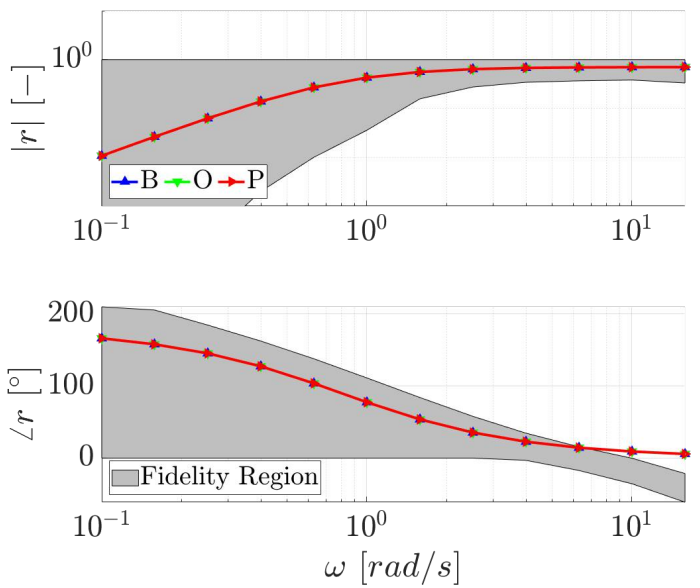

(c) Test 5
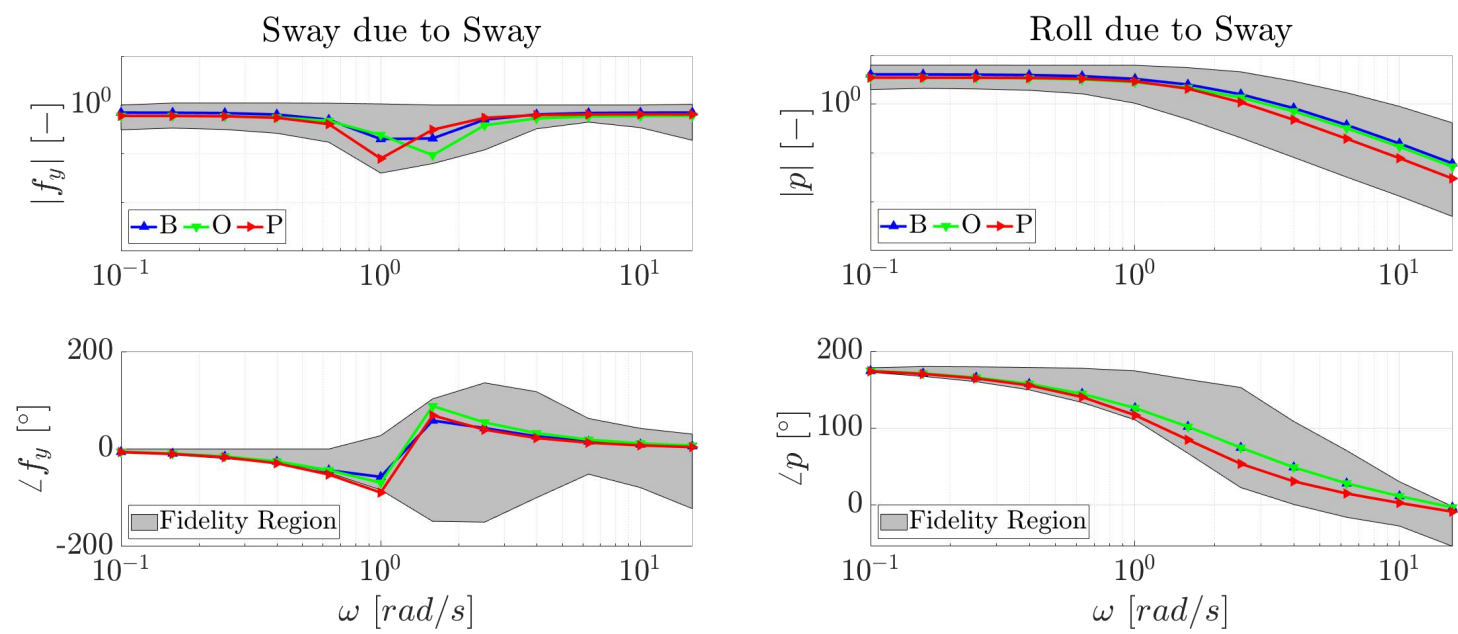

(d) Test 8

(e) Test 9

Fig. 12 Objective Motion Cueing Test Results for all Configurations 
The frequency response for the $P$ configuration performed the worst on the OMCT test. As can be observed, the $P$ configuration lies slightly outside the fidelity boundary for the second half and first half of the roll test 3 and 4 , respectively. Furthermore, it partly lies outside the fidelity boundary of the sway test 8 . This illustrates the fact that the PEMD analysis can yield different results than the OMCT analysis.

Table 4 Classical Washout Algorithm Filter Parameters for all Configurations used in the Experiment

\begin{tabular}{c|cccccccccccc}
\hline \hline & $K_{f_{y}}$ & $\omega_{n_{f_{y}}}$ & $\omega_{b_{f_{y}}}$ & $\zeta_{f_{y}}$ & $\omega_{n_{t}}$ & $\zeta_{t}$ & $K_{p}$ & $\omega_{n_{p}}$ & $\zeta_{p}$ & $K_{r}$ & $\omega_{n_{r}}$ & $\zeta_{r}$ \\
& {$[-]$} & {$[\mathrm{rad} / \mathrm{s}]$} & {$[\mathrm{rad} / \mathrm{s}]$} & {$[-]$} & {$[\mathrm{rad} / \mathrm{s}]$} & {$[-]$} & {$[-]$} & {$[\mathrm{rad} / \mathrm{s}]$} & {$[-]$} & {$[-]$} & {$[\mathrm{rad} / \mathrm{s}]$} & {$[-]$} \\
\hline$B$ & 0.70 & 0.80 & 0.00 & 1.00 & 2.00 & 1.00 & 0.70 & 0.80 & 1.00 & 0.70 & 0.80 & 1.00 \\
$O$ & 0.60 & 1.00 & 0.00 & 1.00 & 2.00 & 1.00 & 0.60 & 0.80 & 0.80 & 0.70 & 0.80 & 1.00 \\
$P$ & 0.60 & 0.75 & 0.00 & 0.911 & 1.50 & 0.80 & 1.067 & 0.928 & 0.70 & 0.70 & 0.80 & 1.00 \\
\hline \hline
\end{tabular}

Table 5 Magnitude- and Phase Distortions for all CWA Configurations

\begin{tabular}{c|cccccc|ccc}
\hline \hline & \multicolumn{7}{|c|}{ Dutch Roll } & \multicolumn{3}{|c}{ Aperiodic Roll } \\
& $\left|f_{y}\right|$ & $\angle f_{y}\left[^{\circ}\right]$ & $|p|$ & $\angle p\left[^{\circ}\right]$ & $|r|$ & $\angle r\left[^{\circ}\right]$ & $\left|f_{y}\right|$ & $|p|$ & $|r|$ \\
\hline$B$ & 0.535 & 13.077 lead & 0.651 & 3.076 lead & 0.687 & 27.929 lead & 0.921 & 0.911 & 1.084 \\
$O$ & 0.393 & 14.768 lead & 0.599 & 0.976 lag & 0.687 & 27.929 lead & 0.836 & 0.882 & 1.084 \\
$P$ & 0.472 & 5.089 lag & 1.010 & 15.305 lead & 0.687 & 27.929 lead & 1.000 & 1.000 & 1.084 \\
\hline \hline
\end{tabular}

\section{B. Participants}

Seven pilots originally participated in the experiment and performed a series of pair-wise comparisons between the three MCA configurations. However, due to problems with the visual system the original Pilot 3 was removed and replaced in the analysis by Pilot 7. Therefore, from now on only six pilots are considered. The order, in which the different motion conditions were evaluated, was designed using a Latin Square distribution. All participating pilots, except Pilot 5, had flying experience with the Cessna Citation 550 aircraft. Pilots 1 to 6 had, at the time of participating, approximately 2,400, 1,300, 3,900, 5,800, 12,600 and 13,000 hours of flight experience, respectively. Also, Pilots 1 and 2 indicated to operate in general aviation, Pilots 3 and 4 indicated to operate in general- and civil aviation, and Pilots 5 and 6 indicated to operate in civil aviation. Furthermore, all pilots, except Pilot 1, deemed to have experience in performing motion simulation experiments. Lastly, all participating pilots were men. This experiment was approved by the Human Research Ethics Committee of the TU Delft, and informed consent was obtained from each pilot.

Table 6 Latin Square Matrix of the Experiment Conditions for all Pilots

\begin{tabular}{|c|c|c|c|c|c|c|c|c|c|}
\hline Pilot & \multicolumn{9}{|c|}{ Conditions } \\
\hline 1 & \multirow{6}{*}{ Training } & $O-P$ & $O-B$ & $P-B$ & \multirow{6}{*}{ 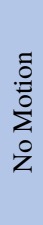 } & $B-P$ & $B-O$ & $P-O$ & \multirow{6}{*}{ 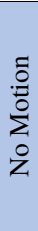 } \\
\hline 2 & & $P-B$ & $O-P$ & $O-B$ & & $B-O$ & $P-O$ & $B-P$ & \\
\hline $3^{*}$ & & $O-B$ & $P-B$ & $O-P$ & & $P-O$ & $B-P$ & $B-O$ & \\
\hline 4 & & $B-P$ & $B-O$ & $P-O$ & & $O-P$ & $O-B$ & $P-B$ & \\
\hline 5 & & $B-O$ & $P-O$ & $B-P$ & & $P-B$ & $O-P$ & $O-B$ & \\
\hline 6 & & $P-O$ & $B-P$ & $B-O$ & & $O-B$ & $P-B$ & $O-P$ & \\
\hline
\end{tabular}

*Pilot 3 has been replaced due to problems with the visual system.

\section{Apparatus}

The SIMONA Research Simulator (SRS) [10], features a hydraulic Stewart motion platform with six degrees of freedom. An impression of the SRS is shown in Figure 13a. It has a collimated display system with a field-of-view of $180^{\circ}$ and $40^{\circ}$, in horizontal- and vertical direction, respectively. Three projectors, mounted on top of the simulator, are used as visual system and have a refresh rate of $120 \mathrm{~Hz}$. FlightGear Flight Simulator was used to render the outside visuals. The 
flight instruments presented to the pilot, Figure $13 \mathrm{~b}$, were rendered using OpenGL. To mask any noise coming from the motion platform, aircraft engine noise was played over the headset.

The simulated aircraft was a nonlinear model of the Cessna Citation 500 business jet. It was trimmed at an altitude of 5,000 m with a weight of 5,207 kg and velocities $V_{T A S}=160 \mathrm{~m} / \mathrm{s}$ and $V_{I A S}=245 \mathrm{kts}$. The aircraft was in cruise configuration with flaps and gear UP and thrust at $94.8 \%$ Fan RPM. It was controlled with the control column and rudder pedals. Finally, the yaw damper and the longitudinal CWA were disabled, so lateral motion cues were clearly felt while longitudinal motion cues were not simulated.

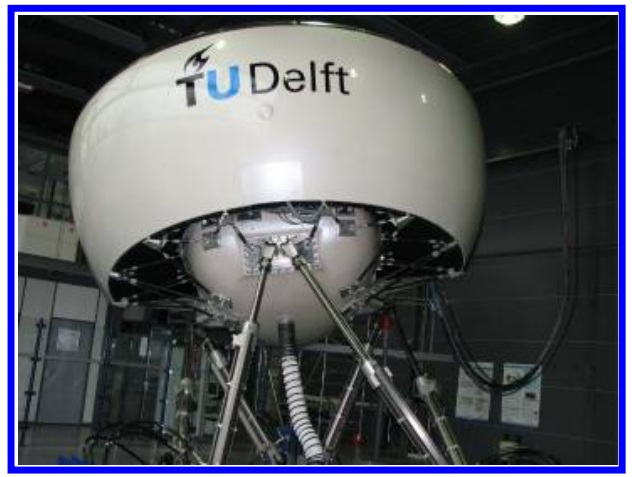

(a) SIMONA Research Simulator

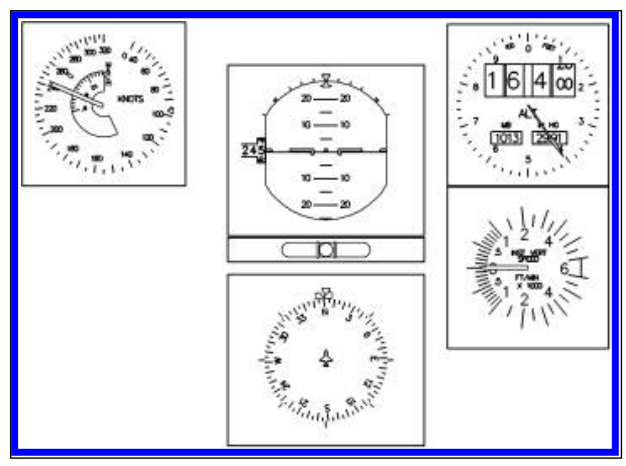

(b) Flight Instruments

Fig. 13 Flight Simulator

\section{Experiment Task}

In the experiment, a series of pair-wise comparisons between the three MCA configurations were performed as a subjective evaluation. This format was based on the experiment by Stoev et al. [9], which received positive feedback. The pilot was instructed to compare pairs of different MCA configurations and select a winner for each pair, which corresponds to the configuration that resembles real flight the most. Due to the subtle differences between configurations and recommendations of Stoev et al. [9], the pilot was asked to give a confidence level for their assessment, ranging from 0 to 2, where; 0 is no confidence, 1 is normal confidence and 2 is high confidence. With a confidence level of 0 , the pair-wise comparison is not taken into account.

Throughout each run, the Dutch roll eigenmode was excited externally with a specially-designed gust of semi-random amplitude and direction. The gust was a single sine-shaped pulse ${ }^{\text {F }}$ with its frequency matching the Dutch roll eigenmode and was injected, every 10 seconds, as a side-slip angle. With this gust, the Dutch roll was excited. However, due to the aircraft's dynamics, the gust also slightly excited the aperiodic roll mode. Within each evaluated pair, one run lasted for 130 seconds and consisted of a passive- and active part.

The first 6 excitations form the passive part, in which the pilot was not allowed to touch the controls. In this part the pilot gets acquainted with the motion signature and natural response of the aircraft, to support their subjective assessment. The last 6 times form the active part, in which the pilot was instructed to suppress the Dutch roll eigenmode at the moment the aircraft was excited. As the aircraft was specially trimmed for the flight condition, as in Table 2, the pilot was instructed to minimize all altitude and heading deviations with respect to the altitude and heading after the passive part. In here the pilot was allowed to use their preferred strategy, i.e., using rudder and/or aileron. As the aircraft was trimmed, the altitude and heading differences between before and after the passive part were negligible.

Besides the 6 pair-wise comparisons between the motion configurations, two additional runs were performed as a no-motion baseline. The aircraft configuration was exactly the same but the MCA was disabled, leaving the motion platform at its neutral position. These runs contained only an active part, which lasted for 130 seconds instead of 60 . One of these runs was after the third pair and one after the sixth. In here, no preferred configuration had to be stated, as this single run was only used to gather objective performance data for the no-motion condition.

\footnotetext{
*Which looks like: $\beta_{g u s t}=\frac{A}{2} \cos \left(\omega_{D R} \cdot t-\pi\right)+\frac{A}{2},\left[0, \frac{2 \pi}{\omega_{D R}}\right]$, where: $A$ and $\omega_{D R}$ are the amplitude and Dutch roll frequency, respectively.
} 


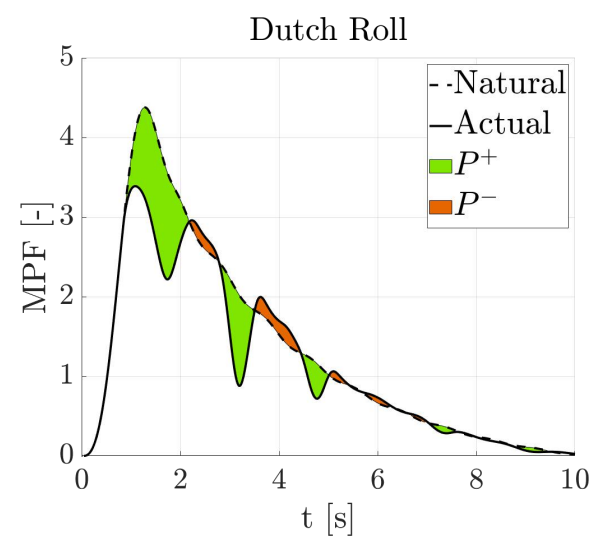

Fig. 14 Visualization of Dutch Roll Suppression Performance

Training was similar to the experiment performed by Stoev et al. [9] and lasted approximately 20 minutes for each pilot, during which the pilot flew all the MCA configurations twice in randomized order based on the Latin Square matrix. Each training run lasted 130 seconds, during which the pilot can practice to suppress the Dutch roll, as well as obtaining a feeling of the aircraft motion signature. Including briefing and training, the experiment lasted for approximately 1.5 hours.

\section{E. Dependent Measures}

The main recorded variables are the preferred MCA configurations in each evaluated pair, i.e., subjective choice, together with a confidence level ranging from 0 to 2 . The preferred configuration is the configuration considered by the pilot to represent the motion cues most realistically.

The influence of motion parameters on performance is tested by determining the Dutch roll suppression performances for each pilot. The Dutch roll MPF's are used for this purpose. Given an external Dutch roll excitation, the dashed line in Figure 14 illustrates the aircraft's natural response, i.e., without pilot input. In the active part, the pilot was instructed to suppress the Dutch roll, giving the actual aircraft response, illustrated by the solid line. Would the actual response lie below the natural response, then this is identified as positive performance $\left(P^{+}\right)$, indicated in green, i.e., green area integrated over time. Contrarily, would the actual response lie above the natural response, then this is identified as negative performance $\left(P^{-}\right)$, indicated in red, i.e., red area integrated over time.

For every 10 seconds, in the active part, the performance $P_{10 s}$ is calculated using Equation 28. In here, $P^{n}$ is the natural response integrated over time. To calculate this performance, the aircraft states and pilot inputs are recorded.

$$
P_{10 s}=\frac{P^{+}-P^{-}}{P^{n}} \cdot 100 \%
$$

\section{F. Hypotheses}

1) It was hypothesized that the PEMD tuned configuration was preferred over the OMCT and baseline tuned configurations, for an aircraft which was excited in the Dutch roll. This was hypothesized as the aircraft dynamics were taken into account during the PEMD tuning process. This hypothesis was rejected if the PEMD tuned configuration received a lower overall score than the OMCT and/or baseline tuned configuration.

2) It was hypothesized that the pilot had a better performance in suppressing the Dutch roll eigenmode with the PEMD tuned configuration than with the OMCT and baseline tuned configurations, as well as in the no-motion condition. This hypothesis was rejected if no significant difference was found, with $\alpha=0.05$.

\section{Results}

Pilot preference for the different motion configurations is analyzed through the results of all pair-wise comparisons. Figure 15 presents the results of all pair-wise comparisons evaluated by all pilots. In here, the bar height represents the number of times a configuration has been preferred, multiplied with the associated confidence level $(0,1$ or 2$)$. Furthermore, each pilot is visualized by a different color. For completion, the raw data are presented in the Table 7. 
Table 7 Pilot Selected Winner for each Pair, with Corresponding Confidence Level

\begin{tabular}{|c|c|c|c|c|c|c|c|c|c|c|c|c|c|c|c|}
\hline Pilot & \multicolumn{15}{|c|}{ Conditions } \\
\hline 1 & \multirow{6}{*}{ Training } & $O-\boldsymbol{P}$ & 1 & $O-B$ & 0 & $P-B$ & 1 & \multirow{6}{*}{$\begin{array}{l}0 \\
0 \\
\sum_{0}^{0} \\
0 \\
0\end{array}$} & B $-P$ & 1 & $B-O$ & 0 & $\boldsymbol{P}-O$ & 1 & \multirow{6}{*}{ 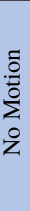 } \\
\hline 2 & & $P-\boldsymbol{B}$ & 1 & $O-P$ & 2 & $O-\boldsymbol{B}$ & 1 & & $B-O$ & 1 & $P-O$ & 2 & $\boldsymbol{B}-P$ & 1 & \\
\hline 3 & & $O-\boldsymbol{B}$ & 2 & $\boldsymbol{P}-B$ & 1 & $O-P$ & 2 & & $P-O$ & 0 & $B-\boldsymbol{P}$ & 2 & $\boldsymbol{B}-O$ & 2 & \\
\hline 4 & & $\boldsymbol{B}-P$ & 1 & $B-O$ & 0 & $\boldsymbol{P}-O$ & 1 & & $O-\boldsymbol{P}$ & 1 & $O-B$ & 0 & $P-B$ & 0 & \\
\hline 5 & & $\boldsymbol{B}-O$ & 2 & $\boldsymbol{P}-O$ & 2 & $\boldsymbol{B}-P$ & 2 & & $P-\boldsymbol{B}$ & 1 & $O-P$ & 1 & $O-\boldsymbol{B}$ & 2 & \\
\hline 6 & & $P-O$ & 1 & $B-\boldsymbol{P}$ & 2 & $B-O$ & 1 & & $O-\boldsymbol{B}$ & 2 & $P-\boldsymbol{B}$ & 2 & $\boldsymbol{O}-P$ & 1 & \\
\hline
\end{tabular}

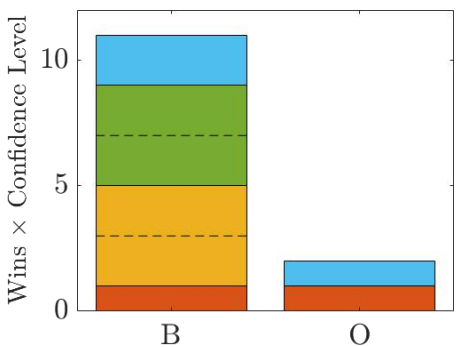

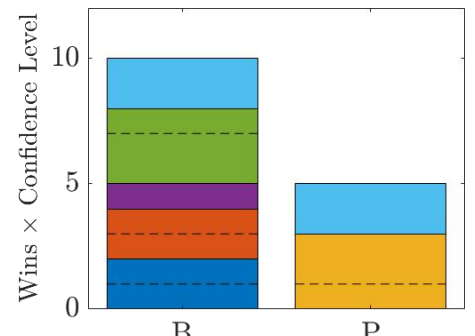

$\mathrm{B}$

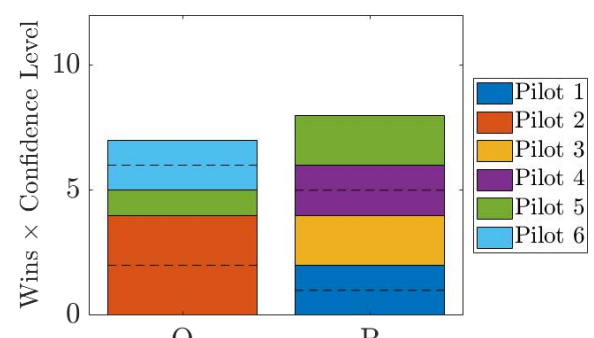

$\mathrm{O}$

$\mathrm{P}$

Fig. 15 Results of Pair-wise Comparisons for Evaluated Pairs

As every pair is evaluated twice per pilot, e.g., $B-O$ and $O-B$, within-pair consistency is present when a pilot prefers the same configuration in both evaluations. Analyzing Figure 15 reveals that Pilot 2 has a within-pair inconsistency in pair $B-O$, Pilot 5 in pair $O-P$, and Pilot 6 in pairs $B-O$ and $B-P$. Not visible from the figure is the number of times that a confidence level of 0 is given. In total, pair $B-O$ received four times a confidence level of 0 , twice by Pilot 1 and 4 . Additionally, pair $B-P$ received once a confidence level of 0 by Pilot 4, and pair $O-P$ once by Pilot 3 .

Statistical modelling is used to make a data-based ranking between the three different configurations. For this, data are fit to a Bradley-Terry pattern model. The Bradley-Terry model is chosen as this model is popular for pair-wise comparisons of highly dependent data [19]. A Matlab implementation ${ }^{\text {f }}$ which uses the Newton-Raphson method, is used to estimate the worth parameters, which provide the probability that configuration $i$ is preferred over $j$, Equation 29

$$
P(i>j)=\frac{\pi_{i}}{\pi_{i}+\pi_{j}}
$$

After normalizing, i.e., $\pi_{B}+\pi_{O}+\pi_{P}=1$, the resulting worth parameters are $\left\{\pi_{B}, \pi_{O}, \pi_{P}\right\}=\{0.601,0.165,0.234\}$. From ranking based on these worth parameters, the $B$ configuration is the winning configuration, with $\pi_{B}=0.601$, followed by configuration $P$ with $\pi_{P}=0.234$. Finally, configuration $O$ with $\pi_{O}=0.165$. Using Equation 29, the probability that configuration $P$ is preferred over configuration $B$ and $O$ is 0.280 and 0.586 , respectively.

This leads to the conclusion that the first hypothesis is rejected, as the $B$ configuration received a higher subjective rating than the $P$ configuration. Yet, the $P$ configuration received a marginally higher rating than the $O$ configuration.

The influence of motion parameters on objectively measured pilot behaviour is tested by determining the Dutch roll suppression performance. This performance is calculated for each pilot for the active parts of each run, resulting in 96 data points per pilot. Which can be divided into 24 data points for each condition, e.g., the three configurations and the no-motion condition. To compare the different conditions, a within-subjects design is used. In a within-subject design, each subject tests all the conditions, yielding one data point for each condition. To fit in within-subject design, the average performance for each pilot and condition is used, i.e., calculating the average of the 24 data points for each pilot and condition. The results, averaged per pilot, for all conditions are presented in Figure 16

"http://personal.psu.edu/drh20/code/btmatlab/ accessed on December 20, 2019 
Performance

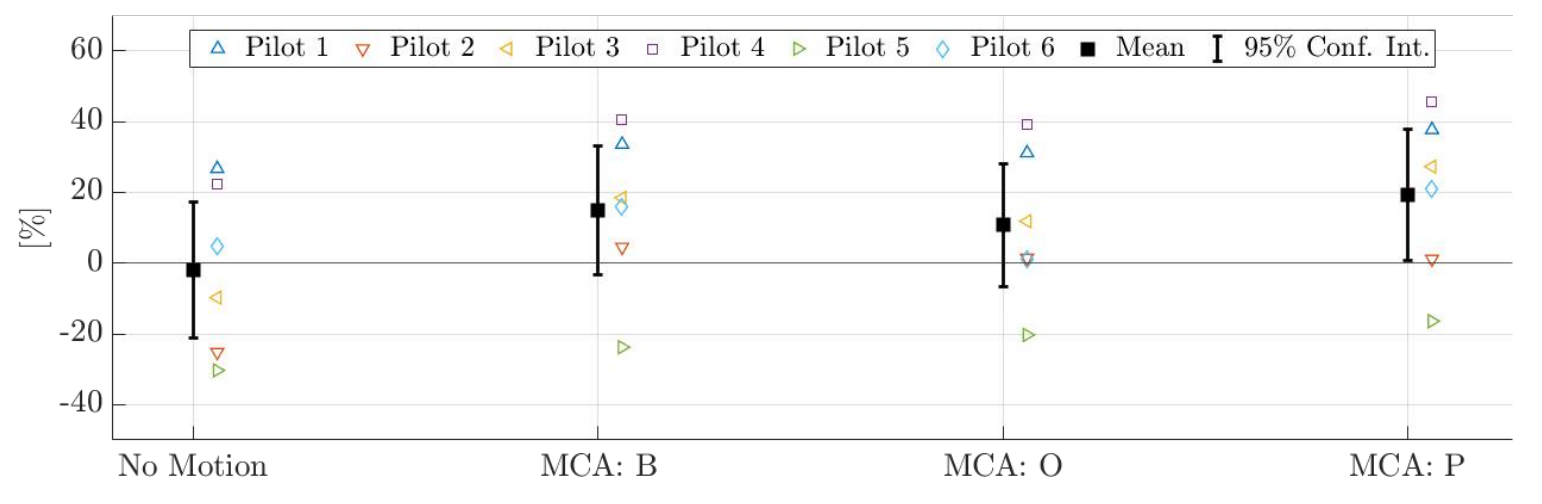

Fig. 16 Performance per Configuration - Not Corrected for Between-Subject Variability

From Figure 16, different observations can be made; Pilots 1 and 4 have the highest performance for all conditions. Pilots 2 and 5 have on average a negative performance, where Pilot 5 even has negative performance for all conditions, meaning that the Dutch roll is excited instead of suppressed. Also, all pilots, except Pilot 6, have lowest performance with the no-motion condition. To correct for between-subject variability, and better observe more general trends over the motion conditions, the data was corrected for individual pilot means, as shown in Figure 17.

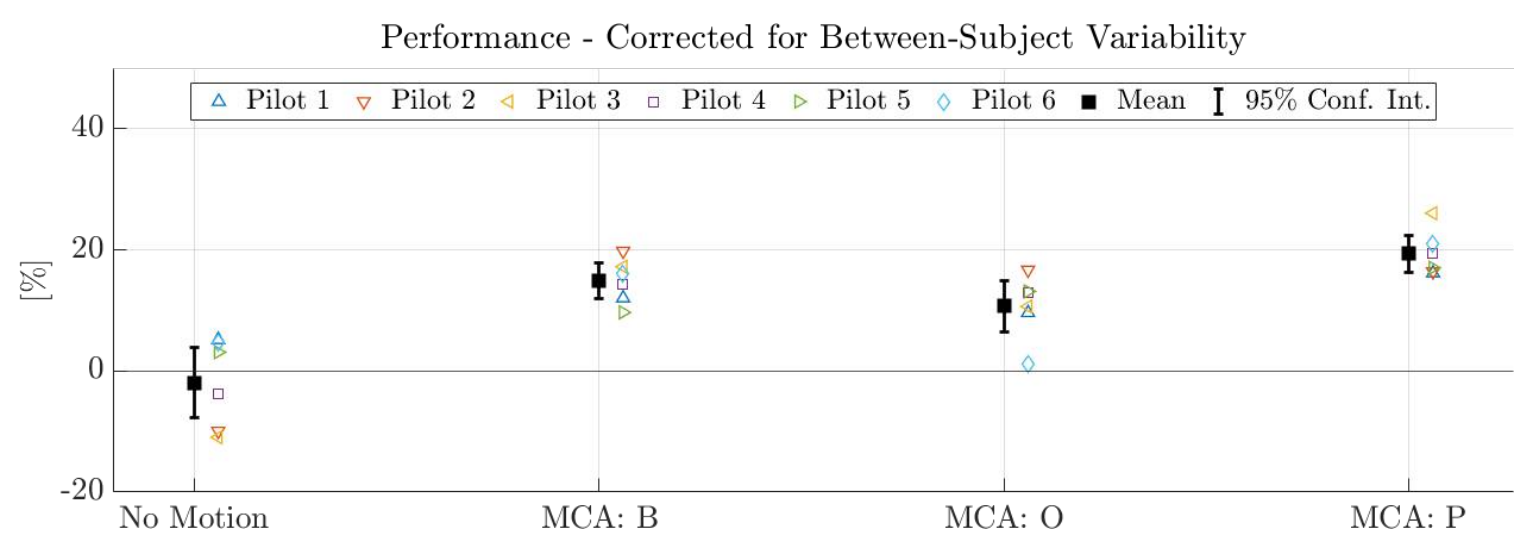

Fig. 17 Performance per Configuration - Corrected for Between-Subject Variability

Comparing Figures 16 and 17 it can be observed that the performances per condition are more concentrated after the between-subject variability correction. Data are concentrated the most for the $B$ - and $P$ configuration. It can be observed that pilots suppressed $19.3 \%$, on average, of the Dutch roll with the $P$ configuration. Furthermore, on average, pilots suppressed $14.9 \%$ and $10.7 \%$ of the Dutch roll with configurations $B$ and $O$, respectively. When the motion was turned-off, pilots were exciting the Dutch roll by approximately $2.0 \%$ instead of suppressing it.

For statistical analysis, a Repeated-Measures ANOVA test is used, as the data were found to satisfy the sphericity- and normality assumption. The results are $F(3,15)=13.832$ and $p<0.0005$, indicating that a significant difference can be identified. Post-hoc tests using the Bonferroni correction, with $\alpha=0.05$, revealed that the pilot performance is significantly better with the $P$ configuration than without motion $(p=0.017)$. However, the improvement in performance between the $B$ configuration and the no-motion condition is not significant ( $p=0.063$ ). Between the $P$ configuration and $B$ configuration no significant difference in performance is found $(p=0.291)$. Furthermore, between the $P$ configuration and $O$ configuration no significant difference is found $(p=0.224)$. The pilots have similar performance with the $B$ and $O$ configurations ( $p=0.966$ ).

The Repeated-Measures ANOVA results are used to test the performance. On average, pilots have the highest performance with the $P$ configuration. A significant improvement is found for the $P$ configuration in comparison to the no-motion 
configuration. Yet, as no significant differences are identified between the $B, O$ and $P$ configurations, the second hypothesis should also be rejected.

\section{Observations}

After performing the experiment the majority of pilots indicated to have difficulties in picking a preferred configuration, as the three configurations felt similar. The pair-wise comparison format used (including confidence levels) received positive feedback. Also, the way each run was divided into a passive- and active part was deemed beneficial for picking a preferred configuration, as the passive part allowed for focus on the motion signature. Also, several pilots suggested to increase the number of pairs to allow for more comparisons as the differences between configurations are very subtle.

Additionally, most pilots indicated deficiencies in the slip indicator. The initial ball direction was correct, but the ball was under-damped. Some pilots even indicated that this influenced their Dutch roll suppression performance. Furthermore, some pilots indicated that the yoke was too sensitive and resulted in over-correction, which sometimes led to exciting the Dutch roll rather than suppressing it.

Different Dutch roll suppression techniques were used by the pilots. First, some pilots only used rudder, whereas others used ailerons, or a combination of both. Second, some pilots gave a single pulse as input, whereas others gave several inputs, e.g. a doublet input. Pilots 1 and 4, which had the highest performance for all conditions, gave mostly doublet inputs to the rudder.

\section{Discussion}

The purpose of this paper is to extend EMD with a pilot perception model, apply the EMD to the lateral model of the Cessna Citation 500, derive an automatic tuning algorithm and validate the resulting motion cueing solution with a pilot-in-the-loop experiment. Six pilots performed pair-wise comparisons of three MCA configurations in an experiment, in which they had to suppress the Dutch roll eigenmode which was externally excited.

The extension of the EMD with a pilot perception model proved useful. Without this extension, the magnitude of the sway specific force is larger, for the Dutch Roll, than of the roll rate. This might be interpreted as the sway specific force being the dominant motion cue in this eigenmode, which is incorrect as a result of the different units. With the pilot perception model, a correct interpretation of the magnitudes can be made, from here it was observed that the roll rate was the dominant motion cue instead.

The implementation of the automatic tuning algorithm in Matlab/Simulink had two difficulties. Firstly, to ensure a consistent calculation of the Euclidean distances, $D_{\square}$, the eigenvectors needed to be scaled properly in each iteration. Secondly, the actuator constraints use a representative manoeuvre, i.e. $\boldsymbol{m}$. This manoeuvre was difficult to implement as it required time domain simulations of the time histories, furthermore it was computationally expensive.

The values for $\alpha$ and $\beta$ in the tuning algorithm were chosen rather arbitrarily. When the Dutch roll is externally excited, the aperiodic roll is excited a little as well. Should this external eigenmode excitation be used in the future, an approach would be to choose values for $\alpha$ and $\beta$ based on the relative MPFs given a certain external excitation.

The $P$ configuration was configured such that the available motion space was similar to the used motion space in configurations $B$ and $O$. Motion cue distortions, associated to the Dutch roll, could have been less if more motion space was available to the tuning algorithm.

Finally, the differences in results for both hypotheses are at first unexpected. The $B$ configuration is the winner in terms of subjective preference. On the other hand, the $P$ configuration seems to be the winner in the objective performance. It must be noted that these two metrics measure slightly different quantities. The first hypothesis is related to perceptual fidelity [11], while the second hypothesis concerns pilot performance. These are two different evaluations and are not necessarily related, e.g., the pilot can perceive something as realistic, but this does not imply that their performance in that condition is good, or even realistic for that matter. 


\section{Recommendations}

In general, EMD/PEMD has shown potential as a motion evaluation and tuning tool. One possible drawback compared to OMCT is that OMCT evaluates fidelity over a range of frequencies, whereas EMD/PEMD only evaluates particular eigenmodes at a certain flight condition. EMD/PEMD tuning might be improved by evaluating eigenmodes corresponding to different flight conditions.

Clearly visible from the results of pair $B-O$ is the need to further refine the OMCT fidelity boundaries. Both configurations satisfy the OMCT fidelity criteria. Among pairs, a confidence level of 0 was given the most to this pair (four times, whereas other pairs only once). Additionally, this pair has two within-pair inconsistencies. This indicates that both configurations are quite similar. Yet, the $B$ configuration performed better on the subjective rating. Furthermore, pilots have a higher suppression performance with this configuration. Both indicate the need for further refinement of the OMCT fidelity boundaries.

Constant perception thresholds are used in the current PEMD. In reality, these vary per person and frequency but also depend on factors such as workload and task [2, 14]. The next step for PEMD could be to use perception thresholds which correspond to the frequency of different eigenmodes. Also, personalized motion cueing can be considered by obtaining someone's perception thresholds and use these to optimize for a motion configuration, which can be useful for training purposes.

In motion cueing, motion is often perceived as too intense if cued one-to-one [20]. This is confirmed by some pilots who indeed reported that perceived motion for the $P$ configuration was too intense. A follow-up study could be to scale the aircraft model associated eigenvectors in the tuning algorithm, which has influence on the relative importance of magnitude and phase distortion minimization. However, less motion space will be necessary.

\section{Conclusion}

This paper presents the application of the Perceptual Eigenmode Distortion applied to a lateral fixed-wing aircraft model. It is shown that by re-expressing the output vector eigenvectors, different units can be compared and the contributions of different motion cues in an eigenmode can be identified. Furthermore, an automatic tuning algorithm was introduced in which an MCA parameter-set can be obtained that minimizes the eigenvector distortions for a given flight condition and representative manoeuvre.

A pilot-in-the-loop experiment was performed for testing the value of the PEMD method. The first hypothesis, which evaluated the pilot preference using pair-wise comparisons, was rejected as the baseline configuration scored highest instead of the PEMD tuned configuration. However, subjective ratings were complicated by pilot inconsistencies and uncertainties. An assessment method that minimizes the pilot bias would be beneficial for future subjective evaluations. Even though pilots have highest performance with the PEMD tuned configuration; the second hypothesis, which evaluated the influence of motion parameters on Dutch roll suppression performance, was rejected as this difference was not significant overall. A significant improvement of the PEMD configuration was only found over the no-motion condition.

In general, PEMD and associated tuning algorithm yield a method for evaluating and tuning motion cueing algorithms. The method has potential and can be used as a stepping stone to further improve objective evaluation methods. Furthermore, the methodology can be applied to any vehicle model and might be used to enhance objective motion cueing as a whole. 


\section{References}

[1] Reid, L. D., and Nahon, M. A., "Flight simulation motion-base drive algorithms: part 1. Developing and testing equations," UTIAS Report, 1985, pp. 5.1-5.24.

[2] Hosman, R. J. A. W., and Van der Vaart, J. C., "Vestibular models and thresholds of motion perception. Results of tests in a flight simulator," Report LR-265, Delft University of Technology, 1978, pp. 26-32.

[3] Sinacori, J. B., "The determination of some requirements for a helicopter flight research simulation facility," NASA, 1977, pp. $1-36$.

[4] Schroeder, J. A., "Helicopter Flight Simulation Motion Platform Requirements," Ph.D. thesis, Stanford University, Jan. 1998.

-[5] Gouverneur, B., Mulder, J. A., Van Paassen, M. M., Stroosma, O., and Field, E., "Optimisation of the SIMONA Research Simulator's Motion Filter Settings for Handling Qualities Experiments," AIAA Modeling and Simulation Technologies Conference and Exhibit, 2003, pp. 25-37. https://doi.org/10.2514/6.2003-5679

[6] Manual of Criteria for the Qualification of Flight Simulation Training Devices - Volume 1 - Aeroplanes, International Civil Aviation Organization, $4^{\text {th }}$ ed., 2015.

[7] Dalmeijer, W., Miletović, I., Stroosma, O., and Pavel, M. D., "Extending the Objective Motion Cueing Test to Measure Rotorcraft Simulator Motion Characteristics," 73rd Annual AHS International Forum and Technology Display, 2017, pp. $1876-1891$.

[8] Miletović, I., "Motion Cueing Fidelity in Rotorcraft Flight Simulation: A New Perspective using Modal Analysis," Ph.D. thesis, Delft University of Technology, Jan. 2020.

[9] Stoev, S., Van Paassen, M. M., Stroosma, O., Miletović, I., and Mulder, M., "Eigenmode Distortion Analysis for Motion Cueing Evaluation in Fixed-Wing Aircraft Simulators," AIAA Modeling and Simulation Technologies Conference and Exhibit, 2019, pp. 1-19. https://doi.org/10.2514/6.2019-0179

- [10] Stroosma, O., Van Paassen, M. M., and Mulder, M., "Using the SIMONA Research Simulator for Human-machine Interaction Research," AIAA Modeling and Simulation Technologies Conference and Exhibit, 2003, pp. 1-8. https://doi.org/10.2514/6.20035525

[11] Pool, D. M., "Objective Evaluation of Flight Simulator Motion Cueing Fidelity Through a Cybernetic Approach,” Ph.D. thesis, Delft University of Technology, Sep. 2012.

[12] Oppenheim, A. V., and Verghese, G. C., Signals, Systems and Inference, Global Edition, Pearson, 2017.

-[13] Heerspink, H., Berkouwer, W. R., Stroosma, O., Van Paassen, M. M., Mulder, M., and Mulder, J. A., "Evaluation of Vestibular Thresholds for Motion Detection in the SIMONA Research Simulator," AIAA Modeling and Simulation Technologies Conference and Exhibit, 2005, pp. 15-17. https://doi.org/10.2514/6.2005-6502.

[14] Groen, E. L., Wentink, M., Valente Pais, A. R., Mulder, M., and Van Paassen, M. M., "Motion Perception Thresholds in Flight Simulation," AIAA Modeling and Simulation Technologies Conference and Exhibit, 2006, pp. 1-11. https: //doi.org/10.2514/6.2006-6254

- [15] De Ridder, K., and Roza, Z. C., "Automatic Optimization of Motion Drive Algorithms using OMCT," AIAA Modeling and Simulation Technologies Conference and Exhibit, 2015, pp. 1-11. https://doi.org/10.2514/6.2015-1139

[16] Fu, W., "Evidence-based development and evaluation of haptic interfaces for manual control," Ph.D. thesis, Delft University of Technology, Jun. 2019. https://doi.org/10.4233/uuid:b5953a17-322d-49e6-87ba-e299673e8b84

[17] Holland, J. H., Adaptation in Natural and Artificial Systems: An Introductory Analysis with Applications to Biology, Control, and Artificial Intelligence, MIT Press, 1992.

[18] Stroosma, O., Van Paassen, M. M., Mulder, M., Hosman, R. J. A. W., and Advani, S. K., "Applying the Objective Motion Cueing Test to a Classical Washout Algorithm," AIAA Modeling and Simulation Technologies Conference and Exhibit, American Institute of Aeronautics and Astronautics, 2013, pp. 1-14. https://doi.org/10.2514/6.2013-4834.

[19] Cattelan, M., "Models for Paired Comparison Data: A Review with Emphasis on Dependent Data," Statistical Science, Vol. 27, No. 3, 2012, pp. 412-433.

-[20] Valente Pais, A. R., Van Paassen, M. M., Mulder, M., and Wentink, M., "Perception Coherence Zones in Flight Simulation," Journal of Aircraft, Vol. 47, 2010, pp. 2039-2048. https://doi.org/10.2514/1.C000281. 